\title{
ON THE REPRESENTATION OF ORDER CONTINUOUS OPERATORS BY RANDOM MEASURES
}

\author{
BY
}

L. WEIS

\begin{abstract}
Using the representation $T f(y)=\int f d \nu_{y}$, where $\left(\nu_{y}\right)$ is a random measure, we characterize some interesting bands in the lattice of all order-continuous operators on a space of measurable functions. For example, an operator $T$ is (lattice-)orthogonal to all integral operators (i.e. all $\nu_{y}$ are singular) or belongs to the band generated by all Riesz homomorphisms (i.e. all $v_{y}$ are atomic) if and only if $T$ satisfies certain properties which are modeled after the Riesz homomorphism property [31] and continuity with respect to convergence in measure. On the other hand, for operators orthogonal to all Riesz homomorphisms (i.e. all $\nu_{y}$ are diffuse) we give characterizations analogous to the characterizations of Dunford and Pettis, and Buhvalov for integral operators. The latter results are related to Enflo operators, to a result of J. Bourgain on Dunford-Pettis operators and martingale representations of operators.
\end{abstract}

1. Introduction. In this paper we consider a kind of operator representation which generalizes the classical form of an integral operator

$$
T f(y)=\int k(y, x) f(x) d \mu(x),
$$

or of a Riesz homomorphism

$$
T f(y)=a(y) \cdot f(\sigma(y))
$$

by replacing the kernel function $y \rightarrow k(y, \cdot) \cdot \mu$ or the point-measures $y \rightarrow a(y) \delta_{\sigma(y)}$ by a more general stochastic kernel. Let $E$ and $F$ be Riesz function spaces over standard measure spaces $(X, \mathscr{A}, \mu)$ and $(Y, \mathscr{B}, \nu)$ respectively (for notations and definitions see below). For every order continuous operator $T: E \rightarrow F$, there is a random measure $\left(\mu_{y}\right)_{y \in Y}$ of measures on $(X, \mathscr{A})$ such that for all $f \in E$

$$
T f(y)=\int f d \mu_{y} \quad \nu \text {-a.e. }
$$

This kind of representation first appeared in probability theory: a regular conditional probability distribution is a representation for a conditional expectation operator. In the context of operator characterizations of Markovian kernels the

Received by the editors January 19, 1983.

1980 Mathematics Subject Classification. Primary 47B55, 45P05, 60G57.

Key words and phrases. Positive operators in function spaces, transition kernels, integral operators, convergence in measure, vector-valued martingales. 
special case $E=F=L_{\propto}$ is given in Neveu's book [36, §5.4]. The case $E=F=L_{2}$ was shown by Arveson [1] who used this representation in his study of operator algebras. For $E=F=L_{1}, \mathrm{~N}$. J. Kalton [19-21] gave an independent proof and applied it to the structure theory of $L_{p}$-spaces and Banach lattices (see also [6]). The general statement (1.3) is due to Sourour [41, 42].

Already in the theory of Markov chains (see [13, Chapter V]), but also in the above applications, it turned out to be useful to decompose $T$ into its "integral" and its "singular" part:

$$
T f(y)=\int f d \nu_{y}^{i}+\int f d \nu_{y}^{s}, \quad \nu_{y}^{i} \ll \mu, \nu_{y}^{s} \perp \mu,
$$

where $\nu_{y}=\nu_{y}^{i}+\nu_{y}^{s}$ is the Lebesgue decomposition with respect to $\mu$, and also into its 'diffuse' and 'atomic' part:

$$
T f(y)=\int f d \nu_{y}^{d}+\int f d \nu_{y}^{a}, \quad \nu_{y}^{d} \text { diffuse, } \nu_{y}^{a} \text { atomic. }
$$

In the lattice of all order continuous operators these decompositions correspond to band decompositions: the integral part of $T$ of course belongs to the band $L_{i}$ of all integral operators (1.1) and the singular part belongs to its orthogonal band $L_{s}$; the atomic part belongs to the band $L_{a}$ generated by all Riesz homomorphisms (1.2) (see Corollary 6.3), and the diffuse part to its orthogonal band $L_{d}$. While there are good characterizations of integral operators and the bands generated by a single Riesz homomorphism [31], the bands $L_{s}, L_{a}, L_{d}$ seem to be less well understood. In this paper we give characterizations of operators in $L_{s}, L_{a}$ or $L_{d}$, which are similar in spirit to the known results on integral operators and Riesz homomorphisms.

For an operator $T$ with a diffuse representation (i.e. $T \in L_{d}$ ), it is clear that (let $d(A)$ be the diameter of a set $A \subset X$ for a metric $d$ on $X$ )

$$
d\left(A_{n}\right) \rightarrow 0 \text { implies } T \chi_{A_{n}} \rightarrow 0 \quad \nu \text {-a.e. }
$$

We show that (1.6) already characterizes such operators-a result analoguous to Buhvalov's characterization of integral operators.

It is well known that an operator $T$ in $L_{1}(X, \mathscr{A}, \mu)$ is an integral operator if and only if

every band $L_{1}(A)$ contains a band $L_{1}(B), A, B \in \mathscr{A}$, such that $T_{\mid L_{1}(B)}$ is compact.

If we replace the band $L_{1}(B)$ in $(1.7)$ by a nonatomic sublattice $L_{1}(\Sigma, \mu)$ ( $\Sigma$ a sub- $\sigma$-algebra of $A$ ), then we obtain a characterization of operators in $L_{d}$. These descriptions of $L_{d}$ together with another one derived from the Dunford-Pettis characterization of integral operators are contained in $\$ 4$.

On the other hand, we can characterize $L_{s}$ and $L_{a}$ (in $\S \S 5$ and 6) by weakening appropriately the disjointness-preserving property of Riesz homomorphisms. For example, $T$ has an atomic representation (i.e. $T \in L_{a}$ ) if for all $L_{1}$-bounded sequences $f_{n} \in E$ we have

$$
t_{n} \stackrel{\mu}{\rightarrow} 0 \text { implies } \quad T f_{n} \stackrel{n^{\prime}}{\rightarrow} 0 .
$$


and $T$ has a singular representation $\left(T \in L_{s}\right)$ if for all such $\left(f_{n}\right)$ we have

$$
d\left(\left\{f_{n} \neq 0\right\}\right) \rightarrow 0 \text { implies } T f_{n} \stackrel{\nu}{\rightarrow} 0 .
$$

Further conditions are inspired by the so-called Maharam property of Luxemburg and Schep [31], and we also express $T \in L_{s}, L_{a}$ in terms of the vector-valued martingale determined by $T^{\prime}$.

In this context we obtain the following generalization of a result of $\mathrm{J}$. Bourgain on Dunford-Pettis operators: If $T: L_{1}[0,1] \rightarrow L_{1}[0,1]$ then $T^{\prime}$ has a diffuse representation if and only if there is a function $G:[0,1] \rightarrow M[0,1]$ such that for $f \in L_{1}[0,1]$

$$
T f=\int G(x) f(x) d \mu(x),
$$

where the Bochner integral exists with respect to the so-called 'discrepancy'-norm on $M[0,1]$ (see Definition 3.8), i.e. $T: L_{1}[0,1] \rightarrow M[0,1]$ is representable with respect to this weaker norm on $M[0,1]$ (see Corollary 4.4 ).

In $\$ 2$ we collect some auxiliary results. In $\$ 3$ we introduce notions which allow us to replace the rather restrictive conditions on diameters in (1.6) and (1.9) by a more general condition.

Some applications of these characterizations to a result of N. Ghoussoub and M. Talagrand on Banach lattices with the Radon-Nikodym property, to convolution operators and to essential spectra of $L_{1}$-operators will appear in [47].

Now we fix some notations and recall some definitions. Let $(X, \mathscr{A}, \mu)$ and $(Y, \mathscr{B}, \nu)$ always be measure spaces where $X$ and $Y$ are separable metric spaces and $\mathscr{A}, \mathscr{B}$ are their $\sigma$-algebras of Borel sets, such that $(X, \mathscr{A})$ and $(Y, \mathscr{B})$ are standard measure spaces [37, Chapter $\mathrm{V}, \S 2$ ]. $\mu$ and $\nu$ always stand for strictly positive and finite measures. $d$ denotes the metric on $X$ or $Y$, and $d(A)$ is the diameter of a subset of $X$ or $Y$. Recall that a random measure from $(Y, \mathscr{B})$ to $(X, \mathscr{A})$ is a family $\left(\mu_{y}\right)_{y \in Y}$ of finite (not necessarily probability-) measures such that for all $A \in \mathscr{A}$ the function $y \in Y \rightarrow \mu_{y}(A)$ is $\mathscr{B}$-measurable. Such a random measure is called $\nu$-orthogonal if all $\mu_{y}$ are probability measures and there is a measurable map $\sigma: X \rightarrow Y$ such that $\mu_{y}\left(\sigma^{-1}(y)\right)=1$ for $\nu$-almost all $y \in Y$. In probability theory these $\nu$-orthogonal random measures appear as regular conditional probability distributions.

$L_{0}(X, \mathscr{A}, \mu)$ is the space of equivalence classes of $\mu$-measurable functions on $X$ and for $A \in \mathscr{A}$ and a $\sigma$-subalgebra $\Sigma$ of $\mathscr{A}$ we use the abbreviations

$$
\begin{gathered}
\mathscr{A}_{A}=\left\{A \cap A^{\prime}: A^{\prime} \in \mathscr{A}\right\}, \quad L_{0}(A, \mu)=L_{0}\left(A, \mathscr{A}_{A},\left.\mu\right|_{\mathscr{A}_{A}}\right), \\
E_{A}=\left\{\left.f\right|_{A}: f \in E\right\}, \quad \Sigma_{A}=\left\{A \cap A^{\prime}: A^{\prime} \in \Sigma\right\}, \\
L_{0}\left(\Sigma_{A}, \mu\right)=L_{0}\left(A, \Sigma_{A}, \sigma_{A},\left.\mu\right|_{\Sigma_{A}}\right), \quad E_{\Sigma}=\{f \in E: f \Sigma \text {-measurable }\},
\end{gathered}
$$

$\mathscr{E}_{\Sigma_{A}}$ denotes the conditional expectation operator from $L_{p}(X, \mathscr{A}, \mu)$ onto $L_{p}\left(\Sigma_{A}, \mu\right)$, $1 \leqslant p \leqslant \infty$.

Let $C(X)$ denote the space of all bounded, continuous functions on $X$ and $M(X)$ the space of all signed, finite measures on $(X, \mathscr{A})$. $\delta_{x}$ is the point measure at $x \in X$. By the Radon-Nikodym theorem we can identify $L_{1}(X, \mu)$ with the subspace 
$\{\lambda \in M(X): \lambda \ll \mu\}$ of $M(X)$ by $f \rightarrow f \cdot \mu$, where $(f \cdot \mu)(A)=\int_{A} f d \mu$. Since $\mu$ is strictly positive we can identify $C(X)$ with a subspace $(C(X))^{\wedge}$ of $L_{\infty}(X, \mathscr{A}, \mu)$ by $g \in C(X) \rightarrow \hat{g} \in L_{\infty}(X, \mathscr{A}, \mu)$. Let $T: L_{\infty}(X, \mathscr{A}, \mu) \rightarrow L_{\infty}(Y, B, \nu)$ be a $w^{*}$-continuous operator with $T\left((C(X))^{\wedge}\right) \subset\left(C(Y)^{\wedge}\right)$. Then $T$ defines an operator $\hat{T}$ : $C(X) \rightarrow C(Y)$ such that $\hat{T}^{\prime}$ is an 'extension' of $T^{\prime}$ in the sense that

$$
\hat{T}^{\prime}(f \cdot \nu)=\left(T^{\prime} f\right) \cdot \mu \text { for } f \in L_{1}(Y, \nu) .
$$

Therefore, in many statements below, we will not distinguish between $f, f \cdot \mu ; C(X)$, $(C(X))^{\wedge}$ and $T, \hat{T}$.

" $\mu_{n} \stackrel{n^{*}}{\rightarrow} \lambda$ " denotes the weak convergence of measures, i.e. the $\sigma(M(X), C(X))$ convergence.

" $f_{n} \stackrel{\mu}{\rightarrow} 0$ " means that $f_{n}$ converges in $\mu$-measure to zero.

By a Riesz function space $E$ over $(X, \mathscr{A}, \mu)$ we mean a sublattice of $L_{0}(X, \mathscr{A}, \mu)$ such that:

(i) $E$ is an ideal in $L_{0}(X, \mathscr{A}, \mu)$,

(ii) $\chi_{A} \in E$ for all $A \in \mathscr{A}$ with $\mu(A)<\infty$.

The Köthe dual of $E$ is given by

$$
E^{\times}=\left\{f \in L_{0}(X, \mathscr{A}, \mu): \int|f \cdot g| d \mu<\infty \text { for all } g \in E\right\} .
$$

If $1 \in E^{\times}$, then $E^{\times}$is a Riesz function space too.

Let $E$ and $F$ be Riesz function spaces and $T: E \rightarrow F$ be a linear operator. We call $T$ regular if for all $f \in E$ there is a $g \in F$ such that $|h| \leqslant f$ implies $|T h| \leqslant g$. The class $L_{r}(E, F)$ of all regular operators is a lattice in the natural ordering $[39, \mathrm{IV}, \S 1 ; 29$; 44, VIII, §2] with the modulus of $T$ given by

$$
|T| f=\sup \{|T g|:|g| \leqslant f\} \quad \text { for } f \in E_{+} .
$$

A regular operator is order continuous if $\left|h_{n}\right| \leqslant f \in E, h_{n} \rightarrow 0$ a.e. always implies that $T h_{n} \rightarrow 0$ a.e. The class $L_{0}(E, F)$ of all order-continuous operators is a band in $L_{r}(E, F)[44$, VIII, § 3]. If $E$ and $F$ are Köthe function spaces with order-continuous norms then every regular operator is order continuous [44, VIII, §3]; if $E$ and $F$ are both abstract $L$-space or order complete abstract $M$-spaces then every bounded operator is regular [39, IV, §1].

Let $E$ and $F$ be Riesz function spaces with $1 \in E^{\times}$and $1 \in F^{\times}$and let $T: E \rightarrow F$ be order continuous. Then the adjoint $T^{\prime}: F^{\times} \rightarrow E^{\times}$is order continuous again.

For unexplained terminology and relevant information on lattices and regular operators see [30, 32, 39], on probability theory see [36, 37], and on Banach spaces see [25-27].

2. The representation theorem. Important special cases of the following result were shown in $[36, \S 5.4 ; 1 ; 19 ; 20 ; 41$ and 12$]$.

2.1 TheOREM [42]. Let $E$ and $F$ be Riesz function spaces over $(X, \mathscr{A}, \mu)$ and $(Y, \mathscr{B}, v)$ respectively. For a regular operator $T \cdot E \rightarrow F$ the following are equivalent:

(a) $T$ is order continuous. 
(b) There is a random measure $\left(\mu_{y}\right)_{y \in Y}$ from $(Y, \mathscr{B})$ to $(X, \mathscr{A})$ such that for all $f \in E$

$$
T f(y)=\int f d \mu_{y} \quad \nu \text {-a.e. }
$$

This representation is unique in the sense that if $\left(\mu_{y}^{\prime}\right)_{y \in Y}$ is a second random measure with $(+)$, then $\mu_{y}=\mu_{y}^{\prime}$ for $\nu$-almost all $y \in Y$.

2.2 REMARK. In $(+)$ it is assumed implicitly that

(i) $\mu(A)=0$ implies $\left|\mu_{y}\right|(A)=0 \nu$-a.e.,

(ii) $\int|f| d\left|\mu_{y}\right|<\infty \nu$-a.e.

Because of (i) we may use the same symbol for a function and its equivalence class.

2.3 Remark [42, Theorem 4.1]. If $S, T: E \rightarrow F$ are order continuous operators and $\left(\mu_{y}[T]\right)$ denotes a representation of $T$ then

(a) $\mu_{y}[T+S]=\mu_{y}[T]+\mu_{y}[S] \nu$-a.e.,

(b) $0 \leqslant T \leqslant S \Leftrightarrow 0 \leqslant \mu_{y}[T] \leqslant \mu_{y}[S] \nu$-a.e.,

(c) $\mu_{y}\left[T^{+}\right]=\mu_{y}[T]^{+}, \mu_{y}[|T|]=\left|\mu_{y}[T]\right| \nu$-a.e.,

(d) $\mu_{y}[T \wedge S]=\mu_{y}[T] \wedge \mu_{y}[S] \nu$-a.e.

The next lemma will be used many times in the following sections. It also provides a proof for Theorem 2.1. (In the following we use identifications of spaces as discussed in (1.11)).

2.4 Lemma. Let $T: E \rightarrow L_{0}(Y, \mathscr{B}, \nu)$ be an order-continuous operator. For every $\varepsilon>0$ there is a compact $K \subset \operatorname{supp} \nu$ with $\nu(Y-K) \leqslant \varepsilon$ such that:

(a) $\chi_{K} T: L_{\infty}(X, \mathscr{A}, \mu) \rightarrow L_{\infty}\left(K, \mathscr{B}_{K}, \nu\right)$ and $\chi_{K} T: C(X) \rightarrow C(K)$.

(b) The operator $\left(\chi_{K} T\right)^{\prime}: L_{1}(K, \nu) \rightarrow L_{1}(X, \mu)$ "extends" to a $w^{*}$-continuous operator $\left(\chi_{K} T\right)^{\prime}: M(K) \rightarrow M(X)$ such that $\left(\chi_{K} T^{\prime}\right)\left(U_{M(K)}\right)$ is uniformly tight.

(c) $\mu_{y}:=\left(\chi_{K} T\right)^{\prime} \delta_{y}, y \in K$, defines a random measure from $\left(K, \mathscr{B}_{K}\right)$ to $(X, \mathscr{A})$ such that

$$
\begin{gathered}
T f(y)=\int f d \mu_{y} \quad \nu \text {-a.e. for all } f \in E, \\
\left(\left(\chi_{K} T\right)^{\prime} \lambda\right)(A)=\int_{K} \mu_{y}(A) d \lambda(y) \quad \text { for } \lambda \in M(K), A \in \mathscr{A} .
\end{gathered}
$$

Proof. (a) $|T|: E \rightarrow L_{0}(Y, \mathscr{B}, \nu)$ exists and is also order continuous. Choose a sequence of compact sets $X_{m} \subset X$ with

$$
X_{m} \subset X_{m+1} \subset \cdots, \quad \mu\left(X-\bigcup_{m} X_{m}\right)=0,
$$

$$
|\mu|_{X_{m}} \text { is strictly positive }
$$

and a sequence $\left(f_{n}\right)$ dense in $\bigcup_{m} C\left(X_{m}\right)$ with respect to the sup-norm. Since $\chi_{X_{m}^{c}} \rightarrow 0$ a.e. it follows that $|T| \chi_{X_{m}^{c}} \rightarrow 0$ a.e.

By Egoroff's theorem and [37, II, Lemma 4.2], there is a compact $K \subset Y$ such that:

$$
\nu(Y-K) \leqslant \varepsilon, \quad K \subset \operatorname{supp} \nu,
$$




$$
\begin{gathered}
|T| 1 \in L_{\infty}(K, \nu), \\
\|\left.\chi_{K}|T| \chi_{X_{m}^{c}}\right|_{\infty} \rightarrow 0 \text { for } m \rightarrow \infty, \\
\text { all }\left.T f_{n}\right|_{K} \text { and }\left.|T| f_{n}\right|_{K} \text { are continuous on } K .
\end{gathered}
$$

By (2.3) the regular operators $T,|T|: L_{\infty}(X, \mu) \rightarrow L_{\infty}(K, \nu)$ are continuous with respect to $\|\cdot\|_{\infty}$-norm. For every $f \in U_{C(X)}$ there is a sequence $\left(f_{n_{m}}\right)$ such that

$$
\left\|T f-T f_{n_{m}}\right\|_{\infty} \leqslant\left\||T| \chi_{X_{m}^{c}}\right\|_{\infty}+\frac{1}{m} \rightarrow 0 \text { for } m \rightarrow \infty .
$$

Therefore, $T: C(X) \rightarrow C(K)$ by (2.5).

(b) The operator $\left(\chi_{K} T\right)^{\prime}: C(K)^{\prime}=M(K) \rightarrow C(X)^{\prime}$ actually takes its values in $M(X)$. Indeed, the finitely additive measure $\left(\lambda_{K} T\right)^{\prime} \lambda, \lambda \in M(K)$, is $\sigma$-additive on $X_{m}$ (cf. [37, II, Theorem 5.7]) and

$$
\left|\left(\chi_{K} T\right)^{\prime} \lambda\right|\left(X_{0}-X_{m}\right)|\leqslant| \lambda\left|\left(|T| \chi_{X_{m}^{c}}\right) \leqslant\|\lambda\| \cdot\left\|\chi_{K}|T|\left(\chi_{X_{m}^{c}}\right)\right\|_{\infty} \stackrel{m \rightarrow \infty}{\rightarrow} 0 .\right.
$$

$\left(\chi_{K} T\right)^{\prime}$ is obviously $w^{*}$-continuous and (2.6) shows that $\left(\chi_{K} T\right)^{\prime}\left(U_{M(K)}\right)$ is uniformly tight. It follows from $\chi_{K} T: L_{\infty}(X, \mu) \rightarrow L_{\infty}(K, \nu)$ and (1.11) that

$$
\lambda \in M(K), \quad \lambda \ll \nu \Rightarrow\left(\chi_{K} T\right)^{\prime} \lambda \ll \mu .
$$

(c) For all $f \in C(X), y \in K$ and $\lambda \in M(X)$ we have

$$
\begin{gathered}
T f(y)=\delta_{y}(T f)=T^{\prime} \delta_{y}(f)=\int f d \mu_{y}, \\
\left(\chi_{K} T\right)^{\prime} \lambda(f)=\lambda\left(\chi_{K} T f\right)=\int_{K} T f(y) d \lambda(y)=\int_{K} \mu_{y}(f) d \lambda(y) .
\end{gathered}
$$

Hence

$$
\left(\chi_{K} T\right)^{\prime} \lambda(A)=\int \mu_{y}(A) d \lambda(y) \text { for all } A \in \mathscr{A} .
$$

By (2.8) the map $y \in K \rightarrow \mu_{y} \in M(X)$ is continuous from $K$ to $M(X)$ with the $w^{*}$-topology. Therefore $\left(\mu_{y}\right)_{y \in K}$ is a random measure such that (cf. (2.7) and (2.9))

$$
\mu(A)=0 \text { implies } \mu_{y}(A)=0 \quad \nu \text {-a.e. }
$$

In order to see that (2.8) holds for $f \in L_{\infty}(X, \mu)$ we choose a sequence $f_{n} \in C(X)$ with $f_{n} \rightarrow f \mu$-a.e. By the order of continuity of $T$ and (2.10) there is a $\nu$-nullset $B$ such that for $y \notin B$

$$
T f_{n}(y) \rightarrow T f(y), \quad f_{n} \rightarrow f \quad \mu_{y} \text {-a.e. }
$$

From (2.8) and the dominated convergence theorem we obtain, for $y \notin B$,

$$
T f(y)=\lim _{n} T f_{n}(y)=\lim \int f_{n} d \mu_{y}=\int f d \mu_{y} .
$$

If $f \in E$ then $f_{n}:=f \wedge n 1 \lambda f, f_{n} \in L_{\infty}$, and (2.8) follows from a similar argument using Fatou's lemma.

2.5 Definition. Let $E$ and $F$ be Riesz iunction spaces over $(X, \mathscr{A}, \mu)$ and $(Y, \mathscr{B}, \nu)$ respectively. Let $L_{i}(E, F)$ be the class of integral operators from $E$ to $F$. 
By $L_{s}(E, F)\left(L_{d}(E, F), L_{a}(E, F)\right)$ we denote the class of all operators with a singular (diffuse, atomic) representation, i.e. the class of all operators $T: E \rightarrow F$ such that $\mu_{y}[T]$ is $\mu$-singular (diffuse, atomic) for $\nu$-almost all $y \in Y$.

2.6 Proposition. $L_{i}(E, F), L_{s}(E, F), L_{d}(E, F)$ and $L_{a}(E, F)$ are bands in $L_{0}(E, F)$ and we have the (lattice) orthogonal decompositions

$$
L_{0}(E, F)=L_{i}(E, F) \oplus L_{s}(E, F), \quad L_{0}(E, F)=L_{d}(E, F) \oplus L_{a}(E, F) .
$$

Proof. The decomposition of a measure $\lambda$ into its diffuse part $\lambda^{d}$ and its atomic part $\lambda^{a}$ as well as the Lebesgue decomposition $\lambda=\lambda^{i}+\lambda^{s}$ into its $\mu$-absolutely continuous part $\lambda^{i}$ and its $\mu$-singular part $\lambda^{s}$ define maps $\lambda \rightarrow \lambda^{d}, \lambda \rightarrow \lambda^{a}, \lambda \rightarrow \lambda^{i}$, $\lambda \rightarrow \lambda^{s}$ in $M(X)$, which are measurable with respect to the Borel structure generated by the $\sigma(M(X), C(X))$-topology on $M(X)$ [9]. Therefore, for a random measure $\left(\mu_{y}\right)_{y \in Y},\left(\mu_{y}^{b}\right)_{y \in Y}$ is again a random measure with $\left|\mu_{y}^{b}\right| \leqslant\left|\mu_{y}\right|, b \in\{d, a, i, s\}$. By Remark 2.3 the required order properties can be reduced to the corresponding order properties of the classes of $\mu$-singular, diffuse or atomic measures in $M(X)$.

Finally we point out a connection between the representation $(+)$ and martingale representations of an operator (cf. [7, Chapter V]).

2.7 Definition. Let $E$ and $F$ be Riesz function spaces over $(X, \mathscr{A}, \mu)$ and $(Y, \mathscr{B}, \nu)$ respectively with $1 \in E^{\times}, 1 \in F^{\times}$. Let $\Sigma_{n}$ be an increasing sequence of algebras, each generated by a finite member of atoms $A_{i}^{n}, i=1, \ldots, s_{n}$, such that $\cup_{n} \Sigma_{n}$ generates $\mathscr{A}$. If $T: E \rightarrow F$ is a linear operator, put

$$
\mathscr{F}_{n}(x)=\sum_{i=1}^{s_{n}} T\left(\chi_{A_{i}^{n}}\right) \cdot \mu\left(A_{i}^{n}\right)^{-1} \cdot \chi_{A_{i}^{n}}(x) .
$$

Then $\left(\mathscr{F}_{n}, \Sigma_{n}\right)$ is a "martingale determined by $T$ ".

2.8 Proposition. Let $T: L_{1}(X, \mathscr{A}, \mu) \rightarrow L_{1}(Y, \mathscr{B}, \nu)$ be bounded and $\left(\mathscr{F}_{n}, \Sigma_{n}\right)$ be a martingale determined by $T$.

(a) For all $g \in L_{\infty}(Y, v)$ we have $T^{\prime} g=L_{1}-\lim g\left(\mathscr{F}_{n}\right)$.

(b) If $\left(\nu_{n}\right)_{x \in X}$ is the representation of $T^{\prime}$, then $w^{*}-\lim \mathscr{F}_{n}(x) \cdot \nu=\nu_{x} \mu$-a.e.

Proof. Since $T$ is $L_{1}$-bounded we have for all $n$

$$
\sup _{x \in X}\left\|\mathscr{F}_{n}(x)\right\|_{L_{1}(Y, v)} \leqslant\|T\|_{L_{1} \rightarrow L_{1}}<\infty
$$

and for all $f \in L_{1}(X, \mu)$ the Bochner integrals $\int_{x} \mathscr{F}_{n}(x) f(x) d \mu(x)$ converge to $T f$ in the $L_{1}(Y, v)$-norm.

(a) For all $g \in L_{\infty}(Y, \nu)$ and $f \in L_{1}(X, \mu)$ we have

$$
\begin{aligned}
T^{\prime} g(f) & =g(T f)=g\left(\lim _{n} \int \mathscr{F}_{n}(x) \cdot f(x) d \mu(x)\right) \\
& =\lim _{n} \int g\left(\mathscr{F}_{n}(x) \cdot f(x)\right) d \mu(x) \\
& =\int\left[\lim _{n} g\left(\mathscr{F}_{n}(x)\right)\right] f(x) d \mu(x)
\end{aligned}
$$

since the scalar-valued martingale $\left(g \circ \mathscr{F}_{n}, \Sigma_{n}\right)$ converges. Hence $T^{\prime} g=\lim _{n} g\left(\mathscr{F}_{n}\right)$. 
(b) Choose a sequence $\left(g_{k}\right) \subset C(Y)$ that generates the $w^{*}$-convergence in $M(Y)$ (cf. [37, II, Theorem 6.6]). Since the martingale $\left(g_{k} \circ \mathscr{F}_{n}, \Sigma_{n}\right)$ converges $\mu$-a.e. to $T^{\prime} g_{k}$ and

$$
\left(T^{\prime} g_{k}\right)(x)=\nu_{x}\left(g_{k}\right) \quad \mu \text {-a.e. for all } k,
$$

there is a $\mu$-nullset $N \in \mathscr{A}$ such that for all $k$ and $x \notin N$

$$
g_{k}\left(\mathscr{F}_{n}(x)\right) \rightarrow \nu_{x}\left(g_{k}\right) \text { for } n \rightarrow \infty .
$$

3. Universal convergence and $U$-equi-integrability. The notions to be introduced in this section are analogous to convergence in measure and equi-integrability respectively, in the sense that "smallness in measure" is replaced by a topological condition.

3.1 Definition. A sequence $A_{n} \in \mathscr{A}$ of Borel sets in $X$ converges to zero universally if every subsequence of $A_{n}$ contains a subsequence $\left(A_{n_{k}}\right)$ such that $\bigcap_{m} \overline{\cup_{k=m}^{\infty} A_{n_{k}}}$ is countable (write $A_{n} \stackrel{u}{\rightarrow} 0$ ).

In particular, if $A_{n} \stackrel{u}{\rightarrow} 0$ then $\mu\left(A_{n}\right) \stackrel{u}{\rightarrow} 0$ for every diffuse measure $\mu$. By [37, Theorem I, 2.8 and II, 8.1], one could also replace the word "countable" in this definition by "universal null set". A "typical" example of a universal nullsequence is the sequence of dyadic intervals $\left[(i-1) / 2^{n}, i / 2^{n}\right], i=1, \ldots, 2^{n}, n \in \mathbf{N}$, or, more generally, every sequence $A_{n} \in \mathscr{A}$ with $d\left(A_{n}\right) \rightarrow 0$. On the other hand, if $A_{n}$ is the set that is taken out of $[0,1]$ at the $n$th step of the construction of the Cantor set, then $A_{n}$ is a pairwise disjoint sequence which does not converge to zero universally.

3.2 Definition. (a) A sequence $\mu_{n} \in M(X)$ goes to zero universally ( $\mu_{n} \stackrel{u}{\rightarrow} 0$ ), if for every $\varepsilon>0$ there is a sequence $A_{n}$ such that $A_{n} \stackrel{u}{\rightarrow} 0$ and $\varlimsup_{\lim _{n}}\left|\mu_{n}\right|\left(A_{n}^{c}\right) \leqslant \varepsilon$.

(b) A subset $U \subset M(X)$ is called $u$-equi-integrable if for all $A_{n} \in \mathscr{A}$ with $A_{n} \stackrel{u}{\rightarrow} 0$ we have $\sup \left\{|\mu|\left(A_{n}\right): \mu \in U\right\} \rightarrow 0$ for $n \rightarrow \infty$.

If all $\mu_{n}$ and $\mu \in U$ are absolutely continuous with respect to a given measure $\nu$ and if one replaces " $A_{n} \stackrel{\leftrightarrow}{\rightarrow} 0$ " by " $\nu\left(A_{n}\right) \rightarrow 0$ " in (a) and (b), then the above conditions become equivalent to convergence in measure and to equi-integrability, respectively. Observe that these definitions depend only on the topology of $X$, but they can also be expressed in terms of the metric given on $X$ :

3.3 Proposition. Let $U$ be a uniformly tight subset of $M_{+}(X)$. Then the following statements are equivalent.

(a) $U$ is u-equi-integrable.

(b) $\sup _{\mu \in U}|\mu|(A) \rightarrow 0$ for $d(A) \rightarrow 0$.

(c) Every limit point of $U$ in the $w^{*}$-topology of $M(X)$ is a diffuse measure.

Proof. (a) $\Rightarrow$ (b) is clear, since $d\left(A_{n}\right) \rightarrow 0$ implies $A_{n} \stackrel{u}{\rightarrow} 0$.

(b) $\Rightarrow$ (c). If $\mu=w^{*}$-lim $\mu_{n}$ with $\mu_{n} \in U$ and if $A_{n}$ is a decreasing sequence of open balls in $X$ with $\cap A_{n}=\{x\}$, then by [37, Theorem II, 6.1]

$$
0 \leqslant \mu(x) \leqslant \mu\left(A_{k}\right) \leqslant \frac{\lim }{n} \mu_{n}\left(A_{k}\right) \rightarrow 0 \text { for } k \rightarrow \infty .
$$


(c) $\Rightarrow$ (a). Otherwise there are sequences $\mu_{n} \in U, A_{n} \in \mathscr{A}$ and a constant $C>0$, such that

$$
\mu_{n}\left(A_{n}\right) \geqslant C, \quad B=\bigcap_{n} \overline{\bigcup_{m=n} A_{m}} \text { is countable. }
$$

Since a uniformly tight subset is $w^{*}$-compact in $M(X)$ [37, Theorem II, 6.7] we may assume $\mu_{n} \stackrel{n^{*}}{\rightarrow} \mu$. For the closed sets $B_{n}=\overline{\bigcup_{n=m}^{\infty} A_{m}}$ we have

$$
\mu\left(B_{m}\right) \geqslant \varlimsup_{n} \mu_{n}\left(B_{m}\right) \geqslant \varlimsup_{n} \mu_{n}\left(A_{n}\right) \geqslant C .
$$

But $\mu(B) \geqslant C$ contradicts the assumption that $\mu$ is a diffuse measure.

3.4 Proposition. Let $\left(\mu_{n}\right) \subset M_{+}(X)$ be a sequence with $\mu_{n} \stackrel{w^{*}}{\rightarrow} \mu \in M(X)$. The following statements are equivalent.

(a) $\mu_{n} \stackrel{u}{\rightarrow} 0$.

(b) For all $\varepsilon>0$ and $\delta_{i}>0, i \in \mathbf{N}$, there are finitely many $K_{i} \in \mathscr{A}$ with $d\left(K_{i}\right)<\delta_{i}$ and $\varlimsup_{n} \mu_{n}\left(X-\cup K_{i}\right) \leqslant \varepsilon$.

(c) $\mu$ is an atomic measure.

Proof. (a) $\Rightarrow$ (c) Choose a subsequence $\left(\mu_{n_{k}}\right)$ and a sequence $A_{k} \in \mathscr{A}$ such that $\varlimsup_{\lim _{k}} \mu_{n_{k}}\left(A_{k}^{c}\right) \leqslant 1 / i$ and $\bigcap_{m} \overline{\bigcup_{k \geqslant m} A_{k}}$ is countable. Since $C_{m}=\overline{\cup_{k \geqslant m} A_{k}}$ is open it follows from the $w^{*}$-convergence of $\mu_{n_{k}}$ [37, Theorem II, 6.1] that

$$
\mu\left(C_{m}\right) \leqslant \frac{\lim }{k} \mu_{n_{k}}\left(C_{m}\right) \leqslant \frac{\lim }{k} \mu_{n_{k}}\left(A_{k}^{c}\right) \leqslant \frac{1}{i} .
$$

For $B_{i}=\bigcup_{m} C_{m}$ we have $\mu\left(B_{i}\right) \leqslant 1 / i$ and $B_{i}^{c}$ is countable. Repeating this argument for all $i \in \mathbf{N}$ we obtain that $\mu$ is zero on $B=\bigcap_{i} B_{i}$ and $B^{c}$ is countable.

(b) $\Rightarrow$ (c). Let $\mu^{d}$ be the diffuse component of $\mu$ and $\varepsilon>0$. Since $\mu^{d}(A) \rightarrow 0$ for $d(A) \rightarrow 0$ there is a sequence $\delta_{i}>0$ such that for all sequences $K_{i}$ with $d\left(K_{i}\right)<\delta_{i}$, $i \in \mathbf{N}$, we have $\mu^{d}\left(\cup_{i \in \mathbf{N}} K_{i}\right) \leqslant \varepsilon$. By assumption, there are finitely many closed $K_{i}$ with $d\left(K_{i}\right)<\delta_{i}$ and $\varlimsup_{n} \mu_{n}\left(X-\bigcup_{i} K_{i}\right) \leqslant \varepsilon$. Using the $w^{*}$-convergence of $\mu_{n}$ we find

$$
\mu^{d}(X) \leqslant \mu\left(X-\cup K_{i}\right)+\mu^{d}\left(\bigcup K_{i}\right) \leqslant \frac{\lim }{n} \mu_{n}\left(X-\cup K_{i}\right)+\varepsilon \leqslant 2 \varepsilon
$$

for all $\varepsilon>0$. Hence $\mu$ is atomic.

(c) $\Rightarrow$ (a). Let $\mu=\sum_{i=1}^{\infty} \alpha_{i} \delta_{x_{i}}$. Given $\varepsilon>0$ we choose an $i_{0}$ such that $\sum_{i=i_{0}}^{\infty} \alpha_{i} \leqslant \varepsilon$. For $A_{m}=\bigcup_{i=1}^{i_{0}} A\left(x_{i}, 1 / m\right)$, where $A\left(x_{i}, 1 / m\right)$ is the open ball with center $x_{i}$ and radius $1 / m$, we obtain

$$
\frac{\lim }{n} \mu_{n}\left(A_{m}\right) \geqslant \mu\left(A_{m}\right)>\sum_{i=1}^{i_{0}-1} \alpha_{i} \geqslant \mu(X)-\varepsilon .
$$

Choose a sequence $\left(n_{m}\right)$ with $n_{m}<n_{m+1}$ so that $\mu_{n}\left(A_{m}\right) \geqslant \mu(X)-\varepsilon$ for $n \geqslant n_{m}$, and put $B_{n}=A_{m}$ if $n_{m} \leqslant n<n_{m+1}$. Then $B_{n} \stackrel{u}{\rightarrow} 0$ and furthermore, we have for $n_{m} \leqslant n<n_{m+1}$

$$
\mu_{n}\left(B_{n}^{c}\right)=\mu_{n}\left(A_{m}^{c}\right) \leqslant \mu_{n}(X)-\mu(X)+\varepsilon
$$

where $\mu_{n}(X)-\mu(X) \rightarrow 0$ for $n \rightarrow \infty$. 
(c) $\Rightarrow$ (b) is shown similarly.

3.5 Proposition. If $\mu_{n} \in M(X)$ is u-equi-integrable and a universal null sequence then $\left\|\mu_{n}\right\|_{M(X)} \rightarrow 0$.

This follows immediately from the definitions. On the other hand we have the following decomposition result whose analogue for convergence in measure and equi-integrability goes back to Kadec and Pekczyński [18, Proof of Theorem 5].

3.6 Proposition. Let $\left(\mu_{n}\right)$ be a bounded, uniformly tight subsequence of $M_{+}(X)$. Then there is a subsequence $\left(n_{k}\right)$ and sequences $\left(\nu_{k}\right),\left(\lambda_{k}\right) \subset M_{+}(X)$ such that:

(i) $\mu_{n_{k}}=\nu_{k}+\lambda_{k}, \nu_{k} \wedge \lambda_{k}=0$.

(ii) $\nu_{k} \stackrel{u}{\rightarrow} 0$ and $\left(\lambda_{k}\right)$ is u-equi-integrable.

Proof. Let $D(\cdot, \cdot)$ be a metric on $U_{M_{+}(X)}$ generating the $\sigma(M(X), C(X))$-topology (cf. [37, Theorem II, 6.2]). Since $\mu_{n}$ is uniformly tight, the sequence is relatively $D$-compact [37, Theorem II, 6.7] and we may assume that $\mu_{n} D$-converges to some $\mu \in M_{+}(X)$. If $\mu^{a}$ and $\mu^{d}$ denotes the atomic part and the diffuse part of $\mu$ respectively, then it is enough to find Borel sets $A_{k}$ and a subsequence $\left(n_{k}\right)$ such that $\left.\mu_{n_{k}}\right|_{A_{k}} \stackrel{D}{\rightarrow} \mu^{a}$. Indeed, according to 3.3 and 3.4, $\nu_{k}=\left.\mu_{n_{k}}\right|_{A_{k}}$ and $\lambda_{k}=\left.\mu_{n_{k}}\right|_{A_{k}^{c}}$ have all the required properties.

Let $\mu^{a}=\sum_{i=1}^{\infty} \alpha_{i} \delta_{x_{i}}$. Since every ball $K$ in $X$ contains a ball $K_{1}$ with $\mu\left(\bar{K}_{1}-\stackrel{\circ}{K}_{1}\right)=0$ (cf. [37, Proof of Lemma II, 6.5]), there is for every $x_{i}$ a decreasing sequence of balls $\left(K_{i}^{m}\right)_{m \in \mathbf{N}}$ with

$$
\left\{x_{i}\right\}=\bigcap_{m} K_{i}^{m}, \quad \mu_{n}\left(K_{i}^{m}\right) \rightarrow \mu\left(K_{i}^{m}\right) \text { for all } m, i .
$$

Given some $\varepsilon>0$ we choose $i_{0}$ such that $D\left(\mu^{a}, \sum_{i=1}^{i_{0}} \alpha_{i} \delta_{x_{i}}\right) \leqslant \varepsilon / 2$. For $B^{m}=\bigcup_{i=1}^{i_{0}} K_{i}^{m}$ we have

$$
\left.\left.\mu_{n}\right|_{B^{m}} \stackrel{D}{\rightarrow} \mu\right|_{B^{m}} \text { for } n \rightarrow \infty
$$

and (by the dominated convergence theorem)

$$
\left.\left.\mu\right|_{B^{m}} \stackrel{D}{\rightarrow} \mu\right|_{\cap_{m} B^{m}}=\sum_{i=1}^{i_{0}} \alpha_{i} \delta_{x_{i}} .
$$

Therefore, there are $m_{0}$ and $n_{0}$ such that $D\left(\left.\mu_{n_{0}}\right|_{B_{0}^{m}}, \sum_{i=1}^{i_{0}} \alpha_{i} \delta_{x_{i}}\right) \leqslant \varepsilon / 2$. Hence for all $\varepsilon>0$ we can find a set $A_{0}$ (put $\left.A_{0}=B^{m_{0}}\right)$ and $n_{0}$ such that $D\left(\left.f_{n_{0}}\right|_{A_{0}}, \mu^{a}\right) \leqslant \varepsilon$. This completes the proof of 3.6 .

3.7 REMARK. We found it convenient to define $u$-convergence in topological terms. From a more 'abstract' point of view the concept can be described as follows: Let $\mathscr{A}$ be a countably generated $\sigma$-algebra of subsets of some set $\Omega$ and let $\mathscr{C} \subset \mathscr{A}$ be a countable generating system, which is closed under finite intersections and unions. Then for $A_{n} \in \mathscr{A}, A_{n} \stackrel{\stackrel{u}{\rightarrow}}{0} 0$ 'means' that every subsequence of $\left(A_{n}\right)$ has a subsequence $\left(A_{n_{m}}\right)$ such that

$$
\bigcap\left\{\in \mathscr{C} \mid \exists k \text { such that } \bigcup_{m=k}^{\infty} A_{n_{m}} \subset C\right\}
$$


is countable. Indeed, by replacing $(\Omega, \mathscr{A})$ by its canonical separable Borel space (cf. [37, V.2]) we may assume $\{x\} \in \mathscr{A}$ for $x \in \Omega$ and then $\mathscr{C}$ is a base of the closed subsets of a metrizable topology on $\Omega$ such that (3.1) corresponds to $\bigcap_{k} \overline{\cup_{m=k}^{\infty} A_{n_{k}}}$ (by Urysohn's metrization theorem [2, p. 125]).

Next we recall a norm on the vector space $M[0,1]$ which is called "discrepancy"norm in mathematical physics (cf. [35]). In the context of representation theory of linear operators it was first used by J. Bourgain [3].

3.8 Definition. $M_{0}$ denotes the vector space $M[0,1]$ normed by $\|\mu\|_{0}=$ $\sup _{I \in J}|\mu(I)|$, where $J$ is the set of all intervals in $[0,1]$.

3.9 REMARK. $M_{0}$ is a noncomplete normed space. If $\left(\mu_{n}\right)$ is bounded in $M[0,1]$ and $\mu_{n} \stackrel{\|\cdot\|_{0}}{\rightarrow} \mu \in M[0,1]$ then $\mu_{n} \stackrel{n^{*}}{\rightarrow} \mu$. If, in addition, all $\mu_{n}$ are diffuse, then so is $\mu$.

3.10 Proposition. Let $U$ be a M[0,1]-bounded set of diffuse measures. If $U$ is u-equi-integrable, then $U$ is relatively compact in $M_{0}$. If all the measures in $U$ are positive then the converse is true too.

Proof. " $\Rightarrow$ " Since $U$ is $w^{*}$-compact it is enough to show that $\mu_{n} \in U, \mu_{n} \stackrel{n^{*}}{\rightarrow} \mu$ implies $\left\|\mu_{n}-\mu\right\|_{0} \rightarrow 0$. Since $\left(\mu_{n}\right)$ is $u$-equi-integrable and $\mu$ is diffuse by Proposition 3.3 there is for every $\varepsilon>0$ some $\delta>0$ such that

$$
d(I)<\delta \Rightarrow|\mu|(I) \leqslant \varepsilon, \quad\left|\mu_{n}\right|(I) \leqslant \varepsilon \text { for all } n .
$$

Furthermore, we can choose finitely many intervals $I_{1}, \ldots, I_{r}$ such that for every interval with $d(I) \geqslant \delta$ there is some $s \in(1, \ldots, r)$ and intervals $J_{1}, J_{2}$ with

$$
I_{s} \Delta I \subset J_{1} \cup J_{2}, \quad d\left(J_{1}\right) \leqslant \delta, d\left(J_{2}\right) \leqslant \delta .
$$

Let $I$ be an arbitrary interval in $[0,1]$. Depending on whether $d(I)<\delta$ or $d(I) \geqslant \delta$ we have by (3.2)

$$
\left|\mu_{n}(I)-\mu(I)\right| \leqslant\left|\mu_{n}\right|(I)+|\mu|(I) \leqslant 2 \varepsilon
$$

or by (3.2) and (3.3)

$$
\left|\mu_{n}(I)-\mu(I)\right| \leqslant 4 \varepsilon+\max _{i=1}^{r}\left|\mu_{n}\left(I_{i}\right)-\mu\left(I_{i}\right)\right| .
$$

Since every interval is a convergence set for the diffuse measure $\mu$, it follows that $\left\|\mu_{n}-\mu\right\|_{0} \leqslant 5 \varepsilon$ for $n$ large enough.

“ $\Leftarrow$ " Let $\varepsilon>0$. By assumption there are finitely many $\mu_{1}, \ldots, \mu_{r}$ with

$$
\min _{i=1}^{r}\left\|\mu-\mu_{i}\right\|_{0} \leqslant \varepsilon \text { for all } \mu \in U .
$$

Therefore there is a $\delta>0$, such that for all $\mu \in U$

$$
\mu(I) \leqslant \varepsilon+\max _{i=1}^{r}\left|\mu_{i}(I)\right| \leqslant 2 \varepsilon \text { for } d(I)<\delta .
$$

Since $\mu \geqslant 0$ the proof is complete.

4. Operators with a diffuse representation. Recall the following characterizations of an integral operator $T: E \rightarrow F$ :

-(Buhvalov [5], [40], [45]) For all $f \in E_{+}$and $A_{n} \in \mathscr{A}$ with $\mu\left(A_{n}\right) \rightarrow 0$ we have $T\left(\chi_{A_{n}} f\right) \rightarrow 0 \nu$-a.e. 
-(Dunford and Pettis [10]) For all $\varepsilon>0$ there is an $A \in \mathscr{A}$ with $\mu\left(A^{c}\right) \leqslant \varepsilon$ such that $T\left(U_{L_{1}(A, \mu)} \cap E\right)$ is equi-integrable.

-(cf. [7, §V.2]) For all $\varepsilon>0$ there is an $A \in \mathscr{A}$ with $\mu\left(A^{c}\right) \leqslant \varepsilon$ such that a martingale determined by $T \chi_{A}$ (see 2.7) converges in $L_{\propto}\left(A, \mu, L_{1}(Y, \nu)\right.$ ).

By replacing convergence in measure, equi-integrability and the $L_{2}\left(L_{1}\right)$-norm by the corresponding 'universal' notions from $\S 3$, we obtain characterizations of operators with a diffuse representation. However, we have to formulate some of the conditions for the adjoint operator. The reason is that it is a self-dual property for a regular operator to be an integral operator, while to have a diffuse representation it is not.

4.1 EXAMPLE. Let $\mu$ be the Lebesgue-measure on $[0,1]$. The operator

$$
T: L_{1}\left([0,1]^{2}, \mu \otimes \mu\right) \rightarrow L_{1}([0,1], \mu), \quad T f(y)=\int f(x, y) d \mu(x)
$$

has the diffuse representation $\mu_{v}=\mu \otimes \delta_{y}$, while $T^{\prime}$ has the atomic representation $\nu_{(x, y)}=\delta_{y}$.

As an example for the $\sigma$-algebra appearing in Theorem 4.2(b), we could choose here the Borel sets of the form $A \times[0,1], A \subset[0,1]$.

4.2 Theorem. Let $E$ and $F$ be Riesz function spaces on $(X, \mathscr{A}, \mu)$ or $(Y, \mathscr{B}, \nu)$ respectively with $1 \in E^{\times}, 1 \in F^{\times}$. For an order continuous operator the following are equivalent.

(a) Thas a diffuse representation.

(b) For all $A \in \mathscr{A}, \mu(A)>0$, there is a $\sigma$-subalgebra $\Sigma$ of $\mathscr{A}_{A}$, such that $\left.\mu\right|_{\Sigma}$ is diffuse and $\left.T\right|_{E_{\Sigma}}$ is an integral operator.

(c) For all $f \in E_{+}$and $A_{n} \in \mathscr{A}$ with $A_{n} \stackrel{u}{\rightarrow} 0$ we have $T\left(\chi_{A_{n}} \cdot f\right) \rightarrow 0$ v-a.e.

(d) For all $\varepsilon>0$ there is a $B \in \mathscr{B}$ with $\nu\left(B^{c}\right) \leqslant \varepsilon$ such that $T^{\prime}\left(U_{l_{1}\left(B, v^{\prime}\right)} \cap F^{\times}\right)$is u-equi-integrable.

(e) (Assume in addition that $X=[0,1]$.) For every $\varepsilon>0$ there is a $B \in \mathscr{B}$ with $\mu\left(B^{c}\right) \leqslant \varepsilon$ such that a martingale determined by $T^{\prime} \chi_{B}: F^{\times} \rightarrow E^{\times}$(see 2.7) converges in $L_{\propto}\left(Y, \nu, M_{0}\right)$ ( see 3.8).

Proof. By 2.4 we can choose compact sets $X_{m} \subset X$ and $Y_{m} \subset Y$ such that:

$$
\begin{aligned}
& X_{m} \subset X_{m+1} \subset \cdots, \quad \mu\left(X-\cup X_{m}\right)=0,\left.\quad \mu\right|_{X_{m}} \text { is strictly positive, } \\
& Y_{m} \subset Y_{m+1} \subset \cdots, \quad \nu\left(Y-\cup Y_{m}\right)=0,\left.\quad \nu\right|_{Y_{m}} \text { is strictly positive, } \\
& \chi_{Y_{n}} T: C(X) \rightarrow C\left(Y_{n}\right), \quad \chi_{X_{m}} T^{\prime}: L_{\infty}(Y, \nu) \rightarrow L_{\infty}\left(X_{m}, \mu\right), \\
& T^{\prime} \chi_{Y_{n}}: L_{1}\left(Y_{n}, \nu\right) \rightarrow L_{1}(X, \mu) \text { 'extends' to a } w^{*} \text {-continuous } \\
& \text { map }\left(\chi_{Y_{n}} T\right)^{\prime}: M\left(Y_{n}\right) \rightarrow M(X), \text { and }
\end{aligned}
$$

$$
T^{\prime}\left(U_{M\left(Y_{n}\right)}\right) \text { is uniformly tight in } M(X) \text { for all } n \text {. }
$$

By $\left(\mu_{v}\right)_{y \in Y}$ we denote the representation of $T$. 
(a) $\Rightarrow$ (b) is essentially shown in [46]: for every random measure $\left(\mu_{y}\right)_{y \in Y}$ with diffuse measures $\mu_{y}$ on $\left(A, \mathscr{A}_{A}\right)$, there is a $\sigma$-subalgebra $\Sigma$ of $\mathscr{A}_{A}$ such that $\mu_{y}$ is $\mu$-absolutely continuous on $\Sigma$ for $\nu$-almost all $y \in Y$.

(b) $\Rightarrow$ (a). If the atomic part of $T$ were not zero, then by (4.3) and [19, Theorem 5.5] there would be an $A \in \mathscr{A}$ such that $T \chi_{A}$ is an isomorphism as an operator from $L_{1}(A)$ to $L_{1}(Y, \nu)$. This contradicts (b).

(a) $\Rightarrow$ (c). For all $y \in Y$ for which $\mu_{y}$ is diffuse and $f$ is $\left|\mu_{y}\right|$-integrable we have $f_{n}=\chi_{A_{n}} f \rightarrow 0$ in $\mu_{y}$-measure and by the dominated convergence theorem

$$
\left|T f_{n}(y)\right|=\left|\mu_{y}\left(f_{n}\right)\right| \rightarrow 0 \text { for } n \rightarrow \infty .
$$

(c) $\Rightarrow$ (d). First we show that $T^{+}$has property (c) too. Given $A_{n} \in \mathscr{A}, A_{n} \stackrel{u}{\rightarrow} 0$, we have for $h_{n}=f \cdot \chi_{A_{n}^{c}}$

$$
\lim \sup T^{+} h_{n} \leqslant T^{+} f \text {. }
$$

On the other hand, for every $0 \leqslant h \leqslant f$ we have $h \wedge h_{n}=h \cdot \chi_{A_{n}^{c}}$ and therefore $T\left(h \wedge h_{n}\right) \rightarrow T h \nu$-a.e. This implies

$$
T^{+} f=\sup \{T h: 0 \leqslant h \leqslant f\} \leqslant \liminf T^{+}\left(h_{n}\right) .
$$

From (4.6) and (4.7) we obtain $T^{+} f=\lim T^{+} h_{n}$, i.e. $T^{+}\left(f \cdot \chi_{A_{n}}\right) \rightarrow 0 \nu$-a.e. Hence $T^{+}, T^{-}$and $|T|$ have property (c).

Given $\varepsilon>0$ we choose as $K_{0}$ one of the $Y_{n}$ such that $\nu\left(K_{0}^{c}\right) \leqslant \varepsilon / 2$. From (4.4) we get $\left(\chi_{K_{0}} T\right)^{\prime}: L_{1}\left(K_{0}, \nu\right) \rightarrow L_{1}(X, \mu)$ and by (4.5) we may assume that, in addition to (4.1), the $X_{m}$ satisfy

$$
\sup \left\{\int_{X-X_{m}}|T|^{\prime} f d \mu: f \in U_{L_{1}\left(K_{0}, \nu\right)}\right\} \stackrel{m \rightarrow \infty}{\rightarrow} 0 .
$$

If for every $m$ we find a compact $K_{m} \subset K_{0}$ with $\nu\left(K_{0}-K_{m}\right) \leqslant \varepsilon / 2^{m+1}$ such that

$$
\sup \left\{\int_{A \cap X_{m}}|T|^{\prime} f d \mu: f \in U_{L_{1}\left(K_{m}, \nu\right)}\right\} \rightarrow 0 \quad \text { for } d(A) \rightarrow 0
$$

then $B=\bigcap_{m} K_{m}$ satisfies property (d) because of (4.8), (4.9) and 3.3. For a fixed $X_{m}$ we choose open sets $A_{i}$ and a sequence $i_{k}$ such that

$$
\begin{gathered}
X_{m} \subset \bigcup_{i=i_{k}+1}^{i_{k+1}} A_{i} \text { for all } k, \\
\max \left\{d\left(A_{i}\right) \mid i=i_{k}+1, \ldots, i_{k+1}\right\} \rightarrow 0 \text { for } k \rightarrow \infty .
\end{gathered}
$$

Since $A_{i} \stackrel{u}{\rightarrow} 0$, it follows that $|T| \chi_{A_{i}} \rightarrow 0$ v-a.e. By Egoroff's theorem we find a $K_{m} \subset K_{0}$ with $\mu\left(K_{0}-K_{m}\right) \leqslant \varepsilon / 2^{m+1}$ and

$$
\left\|\chi_{K_{m}}|T| \chi_{A_{i}}\right\|_{\infty} \rightarrow 0 \text { for } i \rightarrow \infty \text {. }
$$

By Lebesgue's covering lemma [22, p. 154] there are $\delta_{k}>0$ such that each $A \subset X_{m}$ with $d(A)<\delta_{k}$ is already contained in one of the sets $A_{i}, i=i_{k}+1, \ldots, i_{k+1}$. 
Therefore, for every $A \subset X_{m}$ with $d(A) \leqslant \delta_{k}$ and $f \in U_{L_{1}\left(K_{m}, v\right)}$ we have

$$
\begin{aligned}
\int_{A}|T|^{\prime}(|f|) d \mu & \leqslant \max _{i=i_{h}+1}^{i_{k+1}} \int_{K_{m}}|f(y)||T|\left(\chi_{A_{i}}\right)(y) d \nu(y) \\
& \leqslant \max _{i=i_{h}+1}^{i_{K+1}}\left\|\chi_{K_{m}}|T| \chi_{A_{i}}\right\|_{\infty} .
\end{aligned}
$$

For $k \rightarrow \infty,(4.9)$ follows from (4.11) and 3.3.

(d) $\Rightarrow$ (a). We may assume that the $Y_{n}$ in addition to (4.2)-(4.4) satisfy

$$
T^{\prime}\left(U_{L_{1}\left(Y_{n}, \nu^{\prime}\right)}\right) \text { is } u \text {-equi-integrable for all } n
$$

(compare (1.11)).

For a fixed $y \in Y_{n}$ we consider the sequence $\nu_{m}=\mu\left(A_{m *}\right)^{-1} \chi_{A_{m}} \cdot \nu$, where $A_{m}$ is a neighborhood base of $y$ in $Y_{n}$. Then $\mu_{m} \stackrel{n^{* *}}{\rightarrow} \delta_{y}$ and $T^{\prime} \mu_{m} \stackrel{w^{* *}}{\rightarrow} T^{\prime} \delta_{y}=\mu_{y}$. Since $\mu_{y}$ is a $w^{*}$-limit point of $T^{\prime}\left(U_{L_{1}\left(Y_{n}, \nu\right)}\right)$ it has to be diffuse by (4.13) and 3.3.

(d) $\Rightarrow$ (f). By (4.4) and (d) there is, for every $\varepsilon>0$, a $B \in \mathscr{B}, \nu\left(B^{c}\right) \leqslant \varepsilon$, such that $V=T^{\prime}\left(U_{L_{1}(B, \nu)}\right)$ is $u$-equi-integrable. By $3.10, V$ is compact as a subset of $M_{0}$. But a martingale $\left(\mathscr{F}_{n}{ }^{\prime}, \Sigma_{n}\right)$ determined by $\left(\chi_{B} T\right)^{\prime}$ takes its values in $V$ and therefore it converges in $M_{0}$.

(f) $\Rightarrow$ (a). If $\left(\mathscr{F}_{n}^{\prime}, \Sigma_{n}\right)$ is determined by $\left(\chi_{B} T\right)^{\prime}$ then $\mathscr{F}_{n}^{\prime}(y) w^{*}$-converges to $\mu_{l}$ for $\nu$-a.e. $y \in B$ according to $2.8(\mathrm{~b})$. Since $\left(\mathscr{F}_{n}^{\prime}, \Sigma_{n}\right)$ converges in $L_{1}\left(B, \nu, M_{0}\right)$ these $w^{*}$-limits are diffuse $\nu$-a.e. in $B$ by the pointwise convergence theorem for martingales and Remark 3.9.

In the characterizations of integral operators quoted above one cannot replace the $L_{1}$-norm by a norm given on $E$. For example, for $E=L_{2}$ there are norm-compact, positive convolution operators which are not integral operators (cf. $[7$, p. 93; 14]). But at least in some cases this can be done for the characterization of diffuse representations.

4.3 Corollary. For a regular operator $T: L_{p}(X, \mathscr{A}, \mu) \rightarrow L_{p}(X, \mathscr{A}, \mu), 1<p<$ $\infty$, the following are equivalent.

(a) $T$ has a diffuse representation.

(b') For all $A \in \mathscr{A}, \mu(A)>0$, there is a $\sigma$-subalgebra $\Sigma$ of $\mathscr{A}_{A}$ such that $\left.\mu\right|_{\Sigma}$ is diffuse and $\left.T\right|_{L_{p}\left(\Sigma_{A}, \mu\right)}$ is norm-compact in $L_{p}$.

$\left(\mathrm{d}^{\prime}\right)$ For all $\varepsilon>0$ there is a $B \in \mathscr{A}$ with $\nu\left(B^{c}\right) \leqslant \varepsilon$ such that $\left\|\chi_{B} T \chi_{{ }_{A}}\right\|_{I_{\cdot, p}} \rightarrow 0$ for $d(A) \rightarrow 0$.

Proof. By [45, Theorem 2.2], there is a density $g \in L_{1}(X, \mu)$ such that (with $\left.\rho_{p}(f)=g^{-1 / p} \cdot f\right)$

$$
T=\rho_{p} T \rho_{p}^{-1}: L_{q}(x, g \cdot \mu) \rightarrow L_{q}(X, g \cdot \mu) \text { for all } 1 \leqslant q \leqslant \infty .
$$

Since $T$ has one of the above properties if and only if $\tilde{T}$ has it we may just as well assume

$$
T: L_{q}(X, \mu) \rightarrow L_{q}(X, \mu) \text { for all } 1 \leqslant q \leqslant \infty .
$$


(a) $\Rightarrow\left(b^{\prime}\right)$. By 4.2 there is a $\sigma$-subalgebra $\Sigma$ of $\mathscr{A}_{A}$ such that $\left.T\right|_{L_{1}(\Sigma, \mu)}$ is an integral operator which we may assume to be compact by [7, §III.2, Proposition 21]. Since $T$ : $L_{\infty}(\Sigma, \mu) \rightarrow L_{\infty}(X, \mu)$ it follows by interpolation [23, Chapter 1, Theorem 3.10], that $\left.T\right|_{L_{p}(\Sigma, \mu)}$ is compact too.

(a) $\Rightarrow\left(\mathrm{d}^{\prime}\right)$. By 4.2 there is for every $\varepsilon>0$ a $B \in \mathscr{A}$ with $\mu\left(B^{c}\right) \leqslant \varepsilon$, such that $T^{\prime} U_{L_{1}(B, \mu)}$ is $u$-equi-integrable, i.e. by 3.3

$$
\left\|\chi_{B} T \chi_{A}\right\|_{L_{\odot}}=\left\|\chi_{A} T^{\prime} \chi_{B}\right\|_{L_{1}} \rightarrow 0 \text { for } d(A) \rightarrow 0 .
$$

By interpolation we have

$$
\begin{aligned}
\left\|\chi_{B} T \chi_{A}\right\|_{L_{-p}} & \leqslant C\left\|\chi_{B} T \chi_{A}\right\|_{L_{\cdot x}}^{1 / p} \cdot\left\|\chi_{B} T \chi_{A}\right\|_{L_{-1}}^{1 / p^{\prime}} \\
& \leqslant C C_{1}\left\|\chi_{B} T \chi_{A}\right\|_{L_{L_{x}}}^{1 / p} \rightarrow 0 \text { for } d(A) \rightarrow 0 .
\end{aligned}
$$

$\left(\mathrm{d}^{\prime}\right) \Rightarrow$ (a). Assume that $T$ has ( $\left.\mathrm{d}^{\prime}\right)$ but not a diffuse representation. By (a) $\Rightarrow\left(\mathrm{d}^{\prime}\right)$ there is a $B \in \mathscr{A}, \mu(B)>0$, such that the atomic part $S$ of $\chi_{B} T$ is nonzero and satisfies $\left\|S \chi_{A}\right\|_{L_{n}} \rightarrow 0$ for $d(A) \rightarrow 0$. But since $S$ is atomic this contradicts the fact that for some $A_{0} \in \mathscr{A}, \mu\left(A_{0}\right)>0, S \chi_{A_{0}}$ is an isomorphism into $L_{p}(X, \mu)$ (use the proof of [19, Theorem 5.4] and observe that by interpolation the claim of Lemma 5.2 in [19] is also true for $L_{p}$-norm).

$\left(b^{\prime}\right) \Rightarrow$ (a) follows similarly to (b) $\Rightarrow$ (a) in 4.2 .

Denote by $I$ and $J$ the operators

$$
\begin{gathered}
I: L_{1}[0,1] \rightarrow C[0,1], \quad I f(y)=\int_{0}^{y} f(z) d \nu(z), \\
J: L_{1}[0,1] \rightarrow M_{0}, \quad J f=f \cdot \nu
\end{gathered}
$$

$(\nu$ is the Lebesgue measure on $[0,1])$. J. Bourgain has shown in $[3$, Theorem 7 and Corollary 8] that for a Dunford-Pettis operator $T: L_{1}[0,1] \rightarrow L_{1}[0,1]$ the operators $I T, J T$ are representable, i.e. there is a function $\mathscr{F} \in L_{\propto}(X, \mu, C[0,1])$ (or $\left.L_{\infty}\left(X, \mu, M_{0}\right)\right)$ such that the Bochner integral

$$
T f=\int_{X} \mathscr{F}(x) f(x) d \mu(x)
$$

exists for all $f \in L_{1}(X, \mathscr{A}, \mu)$ (cf. [7, §III.1]). This result is contained in

4.4 Corollary. For a bounded linear operator $T: L_{1}(X, \mathscr{A}, \mu) \rightarrow L_{1}[0,1]$ the following are equivalent.

(a) IT is representable as in $(*)$.

(b) $J T$ is representable as in $(*)$.

(c) $T^{\prime}$ has a diffuse representation.

A Dunford-Pettis operator has these properties.

Proof. (b) $\Leftrightarrow$ (c) follows from 4.2. (a) $\Leftrightarrow$ (e) with $E=L_{\infty}[0,1], F=L_{\infty}(X, \mathscr{A}, \mu)$ because a martingale $\left(\mathscr{F}_{n}, \Sigma_{n}\right)$ determined by $T$ converges in $L_{\infty}\left(X, \mu, M_{0}\right)$ if and only if $T$ has a representation $(*)$ with $\mathscr{F}=\lim \mathscr{F}_{n}$ (cf. [7, §V.2]). 
(a) $\Leftrightarrow$ (b). For all $f \in L_{1}[0,1]$ and each interval $I=[a, b]$ in $[0,1]$ we have

$$
\left|\int_{I} f(y) d \nu(y)\right| \leqslant\left|\int_{0}^{a} f(y) d \nu(y)\right|+\left|\int_{0}^{b} f(y) d \nu(y)\right| .
$$

Therefore $\|I f\|_{C[0,1]} \leqslant\|J f\|_{M_{0}} \leqslant 2\|J f\|_{C[0,1]}$ and (b) is just a reformulation of (a).

Now we assume that $T$ is a Dunford-Pettis operator such that $T^{\prime}$ does not have a diffuse representation. There is a $B \subset[0,1]$ such that $\left(\chi_{B} T\right)^{\prime}$ can be extended to an operator $L_{1}(B, \nu) \rightarrow L_{1}(X, \mu)$ (let $\left.\chi_{B}|T| 1 \in L_{\infty}[0,1]\right)$ which is even an isomorphism (by [19, Theorem 5.5]). Consequently, if $f_{n} \in L_{\infty}(B)$ is a sequence with $C^{-1} \leqslant\left\|f_{n}\right\|_{L_{1}}$ $\leqslant\left\|f_{n}\right\|_{L_{\infty}} \leqslant C$ and $f_{n} \rightarrow 0$ in $\sigma\left(L_{1}, L_{\infty}\right)$ then $g_{n}=T^{\prime} f_{n}$ has the same properties. We obtain

$$
\left|f_{n}\left(T g_{n}\right)\right|=\left|T^{\prime} f_{n}\left(g_{n}\right)\right|=\int\left|g_{n}\right|^{2} d \mu \geqslant\left\|g_{n}\right\|_{L_{1}}^{2} \geqslant C_{1}^{-2}>0 \text {. }
$$

On the other hand, since $g_{n} \rightarrow 0$ in $\sigma\left(L_{1}, L_{\infty}\right),\left\|g_{n}\right\|_{L_{\infty}} \leqslant C$, it follows from the Dunford-Pettis property of $T$ that

$$
\left|f_{n}\left(T g_{n}\right)\right| \leqslant\left\|f_{n}\right\|_{\infty} \cdot\left\|T g_{n}\right\|_{1} \rightarrow 0 \text { for } n \rightarrow \infty .
$$

This contradiction completes the proof.

4.2(b) together with a result of Enflo and Starbird [11] gives the following alternative (in the following denote by $\Sigma$ or $\Sigma^{\prime}$ a countably generated $\sigma$-subalgebra of algebras of the form $\mathscr{A}_{A}, A \in \mathscr{A}$, such that $\left.\mu\right|_{\Sigma},\left.\mu\right|_{\Sigma^{\prime}}$ are diffuse).

4.5 Corollary. For every bounded linear operator $T: L_{1}(X, \mathscr{A}, \mu) \rightarrow L_{1}(Y, \mathscr{B}, \nu)$ and every $\Sigma$ as above there is either a $\Sigma^{\prime} \subset \Sigma$ such that $\left.T\right|_{L_{1}\left(\Sigma^{\prime}, \mu\right)}$ is an isomorphism or there is a $\Sigma^{\prime} \subset \Sigma$ such that $\left.T\right|_{L_{1}\left(\Sigma^{\prime}, \mu\right)}$ is compact.

Proof. Depending on whether $T: L_{1}(\Sigma, \mu) \rightarrow L_{1}(Y, \nu)$ has a diffuse representation or not we may apply 4.2(b) or [19, Theorem 5.5]. To obtain a criterium that distinguishes the alternatives consider a system of sets $A_{i}^{n} \in \Sigma, i=1, \ldots, 2^{n}, n \in \mathbf{N}$, with

(i) $A_{1}^{n}, \ldots, A_{2^{n}}^{n}$ is a partition of $A$ (if $\Sigma \subset \mathscr{A}_{A}$ ),

(ii) $A_{i}^{n}=A_{2 i-1}^{n+1} \cup A_{2 i}^{n+1}, \mu\left(A_{i}^{n}\right)=2^{-n}$ and

$$
\alpha_{n}:=\left\|\max _{i=1}^{2^{n}}\left|T \chi_{A_{i}^{n}}\right|\right\|_{L_{1}(Y, \nu)} .
$$

If $\alpha_{n} \nrightarrow 0$, then it follows from [11] that $\left.T\right|_{L_{1}\left(\Sigma^{\prime}, \mu\right)}$ is an isomorphism for some $\Sigma^{\prime} \subset \Sigma$. If $\alpha_{n} \rightarrow 0$, use [46] to obtain a $\Sigma^{\prime} \subset \Sigma$ such that $\left.T\right|_{L_{1}\left(\Sigma^{\prime}, \mu\right)}$ is compact.

4.6 Remark. According to Starbird [43] an operator $T: L_{1}(X, \mu) \rightarrow L_{1}(X, \mu)$ is an Enflo operator, if and only if there is a subspace $E$ of $L_{1}(X, \mu)$ isomorphic to $L_{1}(X, \mu)$ such that $\left.T\right|_{E}$ is an isomorphism. From 4.5, [11] and the known characterization of sublattices of $L_{1}$ (cf. [25, §17, Theorem 3]) we obtain

(a) $T$ is not an Enflo operator if and only if every nonatomic sublattice $E$ contains a nonatomic sublattice $F \subset E$ such that $\left.T\right|_{F}$ is compact.

This can be compared with the following known results:

(b) $T$ is an integral operator if and only if each band $E$ of $L_{1}(X, \mu)$ contains a band $F$ such that $\left.T\right|_{F}$ is compact [7, §III.2, Proposition 21]. 
(c) $T$ is weakly compact if and only if every infinite-dimensional subspace $E$ contains an infinite-dimensional subspace $E$ such that $\left.T\right|_{F}$ is compact (Pelczyński [38]).

A further application of $4.2(\mathrm{~b})$ concerning a result of Doss $[8 ; 15,87.5]$ in harmonic analysis is given in [46].

5. Operators with a singular representation. Special examples of operators with a singular representation are Riesz homomorphisms (cf. [39, II.2, III.9; 32, I.7]) and their duals. This is obvious from the following characterizations $\left((A) \Leftrightarrow(C) \Leftrightarrow\left(D^{\prime}\right)\right.$ are well known; see [31, 28, 16]).

5.1 Proposition. Let $E$ and $F$ be Riesz function spaces over $(X, \mathscr{A}, \mu)$ and $(Y, \mathscr{B}, \mu)$ respectively with $1 \in E^{\times}, 1 \in F^{\times}$. For an order-continuous operator $T$ : $E \rightarrow F$ the following are equivalent.

(A) There are measurable maps $\sigma: Y \rightarrow X, a: Y \rightarrow \mathbf{R}$ such that for all $f \in E$

$$
T f(y)=a(y) \cdot f(\sigma(y)) \quad \text { v-a.e. }
$$

(C) $T$ is a Riesz homomorphism, i.e. $|f| \wedge|g|=0$ implies $|T f| \wedge|T g|=0$ for all $f, g \in E$.

(D) For every $\varepsilon>0$ there is a $B \in \mathscr{B}, \nu\left(B^{c}\right) \leqslant \varepsilon$ such that for every sequence $B_{n} \subset B$ with $d\left(B_{n}\right) \rightarrow 0$ there is a sequence $A_{n} \in \mathscr{A}$ with $d\left(A_{n}\right) \rightarrow 0$ and $|T|\left(\chi_{A_{n}}\right) \geqslant \chi_{B_{n}} \cdot|T|(1)$.

(C) For every $\varepsilon>0$ there is a $B \in \mathscr{B}, \nu\left(B^{c}\right) \leqslant \varepsilon$ such that for every sequence $f_{n} \in F_{B}^{\times}$with $d\left(\operatorname{supp} f_{n}\right) \rightarrow 0$ we have $d\left(\operatorname{supp} T^{\prime} f_{n}\right) \rightarrow 0$.

(D') $T^{\prime}: F^{\times} \rightarrow E^{\times}$has the Maharam property [31], i.e. for all $A \in \mathscr{A}$ there is a $B \in \mathscr{B}$ such that $\left|T^{\prime}\right|\left(\chi_{B}\right)=\chi_{A}|T|^{\prime}(1)$.

( $\left.\mathrm{A}^{\prime}\right)$ There is a $\mu$-orthogonal random measure $\left(\nu_{x}\right)_{x \in X}$ on $(Y, B)$ and a measurable $b: Y \rightarrow \mathbf{R}$ such that for all $f \in F^{\times}$

$$
T^{\prime} f(x)=\int_{\sigma^{-1}(x)} f \cdot b d \nu_{x} \quad \text { u-a.e., }
$$

where $\sigma: Y \rightarrow X$ is the separating map belonging to $\left(\nu_{x}\right)_{x \in X}$.

Proof. (C) $\Leftrightarrow\left(\mathrm{D}^{\prime}\right)$. See $[28,31]$.

(C) $\Rightarrow$ (A) is shown in [16] by a lifting argument. Alternatively, if $\left(\mu_{y}\right)_{y \in Y}$ is the representation of $T$ and $A_{n} \in \mathscr{A}$ a sequence that generates $\mathscr{A}$, then there is a $\nu$-nullset $N \subset \mathscr{B}$ such that

$$
A_{n} \cap A_{m}=\varnothing \Rightarrow\left\{y: \mu_{y}\left(A_{n}\right) \neq 0\right\} \cap\left\{y: \mu_{y}\left(A_{m}\right) \neq 0\right\} \subset N .
$$

Therefore, for every $y \in N^{c}$ the support of $\mu_{y}$ is a single point. Otherwise there were $y_{0} \in N^{c}, A_{n_{0}}$ and $A_{m_{0}}$ with $A_{n_{0}} \cap A_{m_{0}}=\varnothing$ and $\mu_{y_{0}}\left(A_{n_{0}}\right)>0, \mu_{y_{0}}\left(A_{m_{0}}\right)>0$ contradicting (5.1). Now choose $\sigma(y)=\operatorname{supp}\left(\mu_{y}\right)$ and $a(y)$ such that $\mu_{y}=a(y) \delta_{\sigma(y)}$. 
(A) $\Rightarrow\left(\mathrm{A}^{\prime}\right)$. Let $\left(\lambda_{x}\right)_{x \in X}$ be the representation of $T^{\prime}$ and $A_{n} \in \mathscr{A}$ a sequence that generates $\mathscr{A}$. For $B_{n}=\sigma^{-1}\left(A_{n}\right)$ we have

$$
\begin{aligned}
\int_{A_{n}}\left|\lambda_{x}\right|\left(Y-B_{n}\right) d \mu(x) & =\left(|T|^{\prime} \chi_{Y-B_{n}}\right) \chi_{A_{n}}=\chi_{Y-B_{n}}\left(|T| \chi_{A_{n}}\right) \\
& =\int \chi_{Y-B_{n}}|a| \cdot \chi_{\sigma^{-1}\left(A_{n}\right)} d \nu=0 .
\end{aligned}
$$

Hence there is a $\mu$-null set $N$ such that

$$
\left|\lambda_{x}\right|\left(Y-B_{n}\right)=0 \text { for all } x \in A_{n}, x \notin N .
$$

For a given $x \notin N$ there is a subsequence $A_{n_{m}}$ with $A_{n_{m}} \supset A_{n_{m} \cdot 1},\{x\}=\bigcap A_{n_{m}}$, $\sigma^{-1}(x)=\bigcap B_{n_{m}}$ so that

$$
\left|\lambda_{x}\right|\left(Y-\sigma^{-1}(x)\right) \leqslant \lim _{m}\left|\lambda_{x}\right|\left(Y-B_{n_{m}}\right)=0 .
$$

Now put $\nu_{x}=\left|\lambda_{x}\right| /\left|\lambda_{x}\right| \mid$ for $\lambda_{x} \neq 0$ and

$$
b(y)=\left\|\lambda_{\sigma(y)}\right\| \cdot\left(\frac{d \lambda_{\sigma(y)}}{\left.d \mid \lambda_{\sigma(y)}\right)}\right)(y) .
$$

$\left(\mathrm{A}^{\prime}\right) \Rightarrow\left(\mathrm{D}^{\prime}\right)$. For a given $A \in \mathscr{A}$ choose $B=\sigma^{-1}(A)$ if $\sigma: Y \rightarrow X$ is the measurable map corresponding to $\left(\nu_{x}\right)_{x \in Y}$.

(A) $\Rightarrow(\mathrm{D})$. Choose a compact $B \in \mathscr{B}, \nu\left(B^{c}\right) \leqslant \varepsilon$, such that $\left.\sigma\right|_{B}$ is continuous. If $d\left(B_{n}\right) \rightarrow 0, B_{n} \subset B$, then $d\left(\sigma\left(B_{n}\right)\right) \rightarrow 0$. Put $A_{n}=\sigma\left(B_{n}\right)$.

(D) $\Rightarrow\left(\mathrm{C}^{\prime}\right)$. Choose a $B$ as in (D). If $B_{n}=\operatorname{supp}\left(f_{n}\right)$ and $d\left(B_{n}\right) \rightarrow 0$ then there are $A_{n}$ with $d\left(A_{n}\right) \rightarrow 0$ and $|T|\left(\chi_{A_{n}}\right) \geqslant \chi_{B_{n}} \cdot|T|(1)$. Therefore

$$
\begin{aligned}
\int|T|^{\prime}\left(\left|f_{n}\right|\right) d \mu & =\int\left|f_{n}\right| \chi_{B_{n}} \cdot|T|(1) d \nu \leqslant \int\left|f_{n}\right| \cdot|T|\left(\chi_{A_{n}}\right) d \nu \\
& =\int_{A_{n}}\left|T^{\prime}\right|\left|f_{n}\right| d \mu .
\end{aligned}
$$

Hence $\operatorname{supp}\left(T^{\prime} f_{n}\right) \subset \operatorname{supp}\left|T^{\prime}\right|\left(\left|f_{n}\right|\right) \subset \overline{A_{n}}$ and $d\left(\overline{A_{n}}\right) \rightarrow 0$.

$\left(\mathrm{C}^{\prime}\right) \Rightarrow(\mathrm{A})$. For every $\varepsilon>0$ there is a compact $B$ as in $\left(\mathrm{C}^{\prime}\right)$ with the additional property that $\left.\nu\right|_{B}$ is strictly positive and $\left(\chi_{B} T\right)^{\prime}: M(B) \rightarrow M(X)$ is defined and $w^{*}$-continuous (by Proposition 2.4). For a fixed $y \in B$ we choose sets $B_{n}$ open in $B$ with $\cap B_{n}=\{y\}$ and $d\left(B_{n}\right) \rightarrow 0$. If $f_{n}=\nu\left(B_{n}\right)^{-1} \chi_{B_{n}}$ then $f_{n} \cdot \nu \stackrel{n^{*}}{\rightarrow} \delta_{y}$ and

$$
\left(T^{\prime} f_{n}\right) \mu=\left(\chi_{B} T\right)^{\prime}\left(f_{n} \cdot \nu\right) \stackrel{n^{* *}}{\rightarrow}\left(\chi_{B} T\right)^{\prime} \delta_{y}=\mu_{y},
$$

where $\left(\mu_{y}\right)_{y \in B}$ is the representation of $\chi_{B} T$. By $\left(\mathrm{C}^{\prime}\right), d\left(\operatorname{supp}\left(T^{\prime} f_{n}\right)\right) \rightarrow 0$ and $\mu_{\mathrm{r}}$, has to be a point measure. Again, put $\sigma(y)=\operatorname{supp} \mu_{1}$ and choose $a(y)$ such that $\mu_{y}=a(y) \cdot \delta_{\sigma(y)}$.

Since every bounded sequence $f_{n} \in L_{1}(\mu)$ with $f_{n} \stackrel{\mu}{\rightarrow} 0$ has a subsequence whose functions are 'almost' pairwise disjoint, the following notion may be considered a weakening of the disjointness preserving property of Riesz homomorphisms and of $\left(\mathrm{C}^{\prime}\right)$ in Proposition 5.1. 
5.2 Definition. Let $E$ and $F$ be Riesz function spaces over $(X, \mathscr{A}, \mu)$ and $(Y, \mathscr{B}, \nu)$, respectively. A linear operator $T: E \rightarrow F$ is called $u$ - $\nu$-continuous, if for every $L_{1}(X, \mu)$-bounded sequence $f_{n} \in E$ with $f_{n} \mu \stackrel{u}{\rightarrow} 0$ we have $T f_{n} \stackrel{\nu}{\rightarrow} 0$.

In the same way condition (D) below may be understood as a weak form of the Maharam property and of (D) in 5.1.

5.3 Theorem. Let $E$ and $F$ be as in 5.2 with $1 \in E^{\times}, 1 \in F^{\times}$. For an order-continuous operator $T: E \rightarrow F$ the following are equivalent.

(A) $T$ has a singular representation.

(B) If $S$ is an integral operator with $0 \leqslant S \leqslant|T|$ then $S=0$.

(C) For all $\varepsilon>0$ there is $a B \in \mathscr{B}$ with $\nu\left(B^{c}\right) \leqslant \varepsilon$ such that $T_{\chi_{B}}$ is $u$-v-continuous.

(D) For all $\varepsilon>0$ there is a $B \in \mathscr{B}, \nu\left(B^{c}\right) \leqslant \varepsilon$, such that for all $B_{n} \in \mathscr{B}_{B}, B_{n} \stackrel{u}{\rightarrow} 0$, and $a>1$ there are $A_{n} \in \mathscr{A}$ with $\mu\left(A_{n}\right) \rightarrow 0$ and

$$
a|T|\left(\chi_{A_{n}}\right) \geqslant \chi_{B_{n}}|T|(1) \text { for all } n \text {. }
$$

(E) For all $\varepsilon>0$ there is an $A \in \mathscr{A}, \mu\left(A_{p}^{c}\right) \leqslant \varepsilon$, such that for a martingale $\left(\mathscr{F}_{n}, \Sigma_{n}\right)$ determined by $T \chi_{A}$ we have $\mathscr{F}_{n}(x) \stackrel{\nu}{\rightarrow} 0$ for $\mu$-almost all $x \in A$.

Furthermore, $T: E \rightarrow F$ has a singular representation if and only if $T^{\prime}: F^{\times} \rightarrow E^{\times}$has one.

Proof. By 2.4 there are compact $X_{n} \subset X, Y_{n} \subset Y$ such that:

$$
\begin{aligned}
& Y_{n} \subset Y_{n+1} \subset \cdots, \quad \nu\left(Y-\cup Y_{n}\right)=0,\left.\quad \nu\right|_{Y_{n}} \text { strictly positive, } \\
& X_{n} \subset X_{n+1} \subset \cdots, \quad \mu\left(X-\cup X_{n}\right)=0,\left.\quad \mu\right|_{K_{n}} \text { strictly positive, } \\
& \chi_{Y_{n}} T, \chi_{Y_{n}}|T|: C(X) \rightarrow C\left(Y_{n}\right), \quad \chi_{Y_{n}}|T|: L_{\infty}(X, \mu) \rightarrow L_{\infty}\left(Y_{n}, \nu\right), \\
& \left(\chi_{Y_{n}} T\right)^{\prime},\left(\chi_{Y_{n}}|T|\right)^{\prime}: L_{1}\left(Y_{n}, \nu\right) \rightarrow L_{1}(X, \mu) \text { extend to } w^{*} \text {-con- } \\
& \text { tinuous operators } M\left(Y_{n}\right) \rightarrow M(X) \text { such that }\left(\chi_{Y_{n}}|T|\right)^{\prime}\left(U_{M\left(Y_{n}\right)}\right) \\
& \text { is uniformly tight, }
\end{aligned}
$$

$$
|T| \chi_{X_{n}}: L_{1}\left(X_{n}, \mu\right) \rightarrow L_{1}(Y, \nu) .
$$

By $\left(\mu_{y}\right)_{y \in Y}$ and $\left(\nu_{x}\right)_{x \in X}$ we denote the representation of $T$ and $T^{\prime}: F^{\times} \rightarrow E^{\times}$ respectively.

(A) $\Leftrightarrow$ (B) follows from 2.3 and 2.6.

Since a regular operator $S$ is an integral operator if and only if $S^{\prime}$ is one, property (B) and therefore property (A) are self-dual.

$(\mathrm{A}) \Rightarrow(\mathrm{C})$. Since $T$ has $(\mathrm{A})$ if and only if $T^{\prime}$ has (A) we may just as well show that " $T$ has (A)" implies " $T$ ' has (C)".

We fix one of the $Y_{n}$ and assume, in addition to (5.2)-(5.5), that all $\mu_{v}, y \in Y_{n}$, are $\mu$-singular. Let $f_{m} \in F_{Y_{n}}^{\times}$be a sequence with $\left\|f_{m}\right\|_{L_{1}\left(Y_{n}, v\right)} \leqslant 1$ and $f_{m} \stackrel{u}{\rightarrow} 0$. It is enough to show that every subsequence of $\left(f_{m}\right)$ has a subsequence $\left(f_{m_{h}}\right)$ such that $T^{\prime} f_{m_{h}} \stackrel{\mu}{\rightarrow} 0$. Hence we may assume that $\left(\left|f_{m}\right| \cdot \nu\right) w^{*}$-converges to some $\lambda \in M_{+}\left(Y_{n}\right)$. By 3.4, $\lambda$ must be an atomic measure of the form $\lambda=\Sigma \alpha_{i} \delta_{y_{i}}$ with $\Sigma\left|\alpha_{i}\right|<\infty, y_{i} \in Y_{n}$. From 
(5.5) and (1.11) we obtain

$$
\left|T^{\prime}\right|\left(\left|f_{m}\right|\right) \cdot \mu=\left|T^{\prime}\right|\left(\left|f_{m}\right| \nu\right) \stackrel{w^{*}}{\rightarrow}\left|T^{\prime}\right| \lambda=\sum_{i} \alpha_{i}\left|T^{\prime}\right| \delta_{y_{i}}=\sum_{i} \alpha_{i}\left|\mu_{y_{i}}\right|
$$

In particular, $\left|T^{\prime}\right| \lambda$ is a $\mu$-singular measure and by Lebesgue's decomposition there is for every $\delta>0$ an open set $A \in \mathscr{A}$ with $\mu(A) \leqslant \delta$ and $\left|T^{\prime}\right| \lambda(X-A)=0$. Since $X-A$ is closed it follows from (5.7) that

$$
\varlimsup_{m} \int_{A^{c}}\left|T^{\prime}\right|\left(\left|f_{m}\right|\right) d \mu \leqslant\left|T^{\prime}\right| \lambda(X-A)=0
$$

i.e. $\left|T^{\prime} f_{m}\right| \leqslant\left|T^{\prime}\right|\left(\left|f_{m}\right|\right) \stackrel{\mu}{\rightarrow} 0$ for $m \rightarrow \infty$.

$(\mathrm{C}) \Rightarrow(\mathrm{E})$. Let $\left(\mathscr{F}_{n}, \Sigma_{n}\right)$ be a martingale determined by $T \chi_{A}$ with the additional property

$$
\sup \left\{d(C): C \text { atom of } \Sigma_{n}\right\} \rightarrow 0 \text { for } n \rightarrow \infty \text {. }
$$

If $x \in A$ is contained in the atoms $C_{n} \in \Sigma_{n}$, then $f_{n}=\mu\left(C_{n}\right)^{-1} \chi_{C_{n}} \stackrel{u}{\rightarrow} 0$ and $\mathscr{F}_{n}(x)=$ $T\left(f_{n}\right) \stackrel{\mu}{\rightarrow} 0$ if $A$ is chosen as in (C).

$(\mathrm{E}) \Rightarrow(\mathrm{B})$. A martingale $\left(\mathscr{F}_{n}, \Sigma_{n}\right)$ determined by an integral operator $S: L_{1}(\mu) \rightarrow$ $L_{1}(\nu)$ converges pointwise $\mu$-a.e. to the kernel function $x \rightarrow k(\cdot, x)$ of $S$ (cf. [7, $\S$ V.2]). If $0 \leqslant S \leqslant|T|$ it follows that $\mathscr{F}_{n}(x) \stackrel{\nu}{\rightarrow} 0$, i.e. $S=0$.

In $(C) \Rightarrow(E)$ only martingales with the additional property (5.8) are considered. Therefore we add

(A) $\Rightarrow$ (E). If $T$ has a singular representation then so does $|T|^{\prime}$. Choose $A=X_{n}$ such that $\mu\left(A^{c}\right) \leqslant \varepsilon$ and $T \chi_{A}: L_{1}(A, \mu) \rightarrow L_{1}(Y, \nu)$. For a martingale $\left(\mathscr{G}_{n}, \Sigma_{n}\right)$ determined by $|T| \chi_{A}$ we have by Proposition 2.8

$$
\mathscr{G}_{n}(x) \cdot \nu \stackrel{w^{*}}{\rightarrow}\left|\nu_{x}\right| \quad \mu \text {-a.e. in } A .
$$

Fix an $x$ with (5.9) and $\left|\nu_{x}\right| \perp \nu$. By Lebesgue's decomposition there is for every $\delta>0$ an open set $B$ in $Y$ such that $\left|\nu_{x}\right|(Y-B)=0$ and $\nu(B) \leqslant \delta$. From (5.9) we obtain

$$
\varlimsup_{n} \int_{Y-B}\left|\mathscr{G}_{n}(x)\right| d \nu \leqslant\left|\nu_{x}\right|(Y-B)=0 .
$$

Hence $\mathscr{G}_{n}(x) \stackrel{\nu}{\rightarrow} 0$ for $\mu$-almost all $x \in A$. The same is true for a martingale $\left(\mathscr{F}_{n}, \Sigma_{n}\right)$ determined by $T \chi_{A}$ because $\left|\mathscr{F}_{n}(x)\right| \leqslant \mathscr{G}_{n}(x)$.

$(\mathrm{A}) \Rightarrow(\mathrm{D})$. Given $\varepsilon>0$ and $a>1$ choose sequences $\varepsilon_{n}>0$ with $\sum_{n=1}^{\infty} \varepsilon_{n}<\varepsilon$, $a_{i}>1$ with $a_{n}<a_{n+1}<\cdots<a$ and a $\delta_{n}>0$ with $\delta_{n} \rightarrow 0$.

First we construct systems of sets $M_{i}^{n} \in \mathscr{A}, N_{i}^{n} \in \mathscr{B}, i=1, \ldots, s_{n}$, and $K_{n} \in \mathscr{B}$ such that for $n>1$ :

The $M_{i}^{n}, i=1, \ldots, s_{n}$, are closed in $X$ with $\mu\left(M_{i}^{n}\right) \leqslant \delta_{n}$,

$$
\text { The } N_{i}^{n}, i=1, \ldots, s_{n} \text {, are open in } K_{n} \text { and } \bigcup_{i=1}^{s_{n}} N_{i}^{n}=K_{n} \text {, }
$$


Every $N_{i}^{n}$ is contained in some $N_{j}^{n-1}$ and then $M_{i}^{n}$ is contained in $M_{j}^{n-1}$,

$$
a_{n} \cdot|T|\left(\chi_{M_{i}^{n}}\right) \geqslant \chi_{N_{i}^{n}}|T|(1) \quad \nu \text {-a.e., }
$$

$K_{n}$ is compact with $K_{n} \subset K_{n-1}, \nu\left(K_{n-1}-K_{n}\right) \leqslant \varepsilon_{n}$, and we have $\chi_{K_{n}} T \chi_{M_{i}^{n-1}}: C\left(M_{j}^{n-1}\right) \rightarrow C\left(K_{n}\right)$ for $i=1, \ldots, s_{n-1}$.

To start we choose a compact $K_{1} \subset Y$ with $\mu\left(K_{1}^{c}\right) \leqslant \varepsilon_{1}$ such that all $\mu_{y}, y \in K_{1}$, are $\mu$-singular and put $s_{1}=1, M_{1}^{1}=X, N_{1}^{1}=K_{1}$. Assume now that all $K_{m}, M_{i}^{m}, N_{i}^{m}$, $m<n$, are already constructed. By 2.4 we can find a $K_{n}$ satisfying (5.14). Choose some $1<b_{1}<b$ with $b \cdot a_{n-1}<a_{n}$. For a fixed $y \in K_{n} \cap N_{i}^{n-1}$ we use the abbreviations $N=N_{i}^{n-1} \cap K_{n}, M=M_{i}^{n-1}$. Since $\mu_{y}$ and $\mu$ are disjoint on $M$ there is (by [39, II, Proposition 4.3]) a sequence of continuous functions $h_{n}$ on $M$ with

$$
0 \leqslant h_{j} \leqslant 1, \quad \mu\left(h_{j}\right) \rightarrow 0, \quad\left|\mu_{y}\right|\left(h_{j}\right) \stackrel{j}{\rightarrow}\left|\mu_{y}\right|(M) .
$$

In particular, there is a $j_{0}$ such that

$$
\begin{gathered}
\mu\left(M_{y}\right)<\delta_{n} \text { for } M_{y}=\left\{h_{j_{0}} \geqslant \frac{b-b_{1}}{b \cdot b_{1}}\right\}, \\
\left(|T| \chi_{M}\right)(y)=\left|\mu_{y}\right|(M)<b_{1}\left|\mu_{y}\right|\left(h_{j_{0}}\right)=b_{1}\left(|T| h_{j_{0}}\right)(y) .
\end{gathered}
$$

Since $|T| \chi_{M}$ and $|T| h_{j_{0}}$ are continuous on $K_{n}$ there is an open neighborhood $N_{y}$ of $y$ in $K_{n}$, such that (5.16) holds for all $z \in N_{y}$. From (5.16) we obtain, for $v$-almost all $z \in N_{y}$,

$$
\begin{aligned}
b_{1}\left(|T| \chi_{M_{y}}\right)(z) & \geqslant b_{1}|T|\left(h_{j_{0}}\right)(z)-b_{1}|T|\left(h_{j_{0}} \chi_{M-M_{y}}\right)(z) \\
& \geqslant|T|\left(\chi_{m}\right)(z)-b_{1}|T|\left(\frac{b-b_{1}}{b b_{1}} \chi_{M}\right)(z)=\frac{b_{1}}{b}|T|\left(\chi_{m}\right)(z) .
\end{aligned}
$$

Since $b \cdot a_{n-1}<a_{n}$ and by (5.13) for $M$ and $N$ we get

$$
a_{n}\left(|T| \chi_{M_{y}}\right)(z) \geqslant a_{n-1}|T|\left(\chi_{M}\right)(z) \geqslant \chi_{N_{y}}|T|(1)(z)
$$

for $\nu$-almost all $z \in N_{y} \subset N$. We can find such $N_{y}$ and $M_{y}$ for all $y \in K_{n}$. Since $K_{n}$ is compact there are finitely many $N_{y_{1}}, \ldots, N_{v_{s_{n}}}$ that cover $K_{n}$. Put $N_{i}^{n}=N_{y_{i}}$ and $M_{i}^{n}=M_{y_{i}}, i=1, \ldots, s_{n}$. This completes the construction of $N_{i}^{n}, M_{i}^{n}, K_{n}$ with (5.10) to (5.14). Finally define $B=\bigcap_{n} K_{n}$. By (5.14) we have $\nu\left(B^{c}\right) \leqslant \Sigma \varepsilon_{n}<\varepsilon$.

Let $B_{k} \in \mathscr{B}_{B}$ be a sequence with $B_{k} \stackrel{u}{\rightarrow} 0$. We may assume without loss of generality that the $B_{k}$ are open in $B$. Indeed, for $\tilde{B}_{k}=\left\{y \in B: d\left(B_{k}, y\right)<1 / k\right\}$ we have for all subsequences $\left(k_{l}\right)$

$$
\bigcap_{m} \overline{\bigcup_{l=m}^{\infty} B_{k_{l}}}=\bigcap_{m} \overline{\bigcup_{l=m}^{\infty} \tilde{B}_{k_{l}}}
$$

Define $A_{k}=\bigcup\left\{M_{i}^{n}: N_{i}^{n} \subset B_{k}\right\}$. From (5.11) and (5.12) it follows that $a \cdot|T|\left(\chi_{A_{k}}\right) \geqslant$ $\chi_{B_{k}}(T 1) \nu$-a.e. and it remains to show that $\mu\left(A_{k}\right) \rightarrow 0$. Otherwise there would be a subsequence $k_{l}$ such that $\mu\left(A_{k_{l}}\right) \geqslant \delta>0$. Since $B_{k} \stackrel{u}{\rightarrow} 0$ we may assume that 
$C=\bigcap_{m} C_{m}, C_{m}=\overline{\bigcup_{l=m}^{\infty} B_{k_{l}}}$, is countable. Therefore, for a sequence $\left(n_{j}\right)$ with $\sum \delta_{n_{j}}<$ $\delta / 2$, the countable set $C$ is contained in a set of the form $N_{0}=\bigcup_{j} N_{i}{ }_{i}{ }_{j}$. Observe that for $M_{0}=\bigcup_{j} M_{i}{ }_{i}{ }_{j}$ we have by (5.10) $\mu\left(M_{0}\right) \leqslant \Sigma \delta_{n_{j}}<\delta / 2$. On the other hand, since $N_{0}^{c} \cap \cap C_{m}=\varnothing$ and all the sets involved are compact, there is an $m_{0}$ such that $C_{m_{0}} \subset N_{0}$. But $B_{k_{m_{0}}} \subset C_{m_{0}} \subset N_{0}$ implies $A_{k_{m_{0}}} \subset M_{0}$ by (5.12) and the definitions of $A_{k}$. This contradicts $\mu\left(A_{k_{m_{0}}}\right)>\delta$ and therefore we showed that $\mu\left(A_{k}\right) \rightarrow 0$.

(D) $\Rightarrow(\mathrm{B})$. Given $0 \leqslant S \leqslant|T|$ and $\varepsilon>0$ there is a $B \in \mathscr{B}, \nu\left(B^{c}\right) \leqslant \varepsilon$, such that $|T|: L_{\infty}(X, \mu) \rightarrow L_{\infty}(B, \nu)$ has (D) and $S: L_{\infty}(X, \mu) \rightarrow L_{\infty}(B, \nu)$ is a compact integral operator (cf. [7, §V.2, Proposition 21]) with (D). Choose a sequence $B_{n} \in \mathscr{B}_{B}$ with $d\left(B_{n}\right) \rightarrow 0$ and $\left\|\chi_{B_{n}} S 1\right\|_{\infty} \geqslant \frac{1}{2}\left\|\chi_{B} S 1\right\|_{\infty}$. By (D) there are $A_{n} \in \mathscr{A}, \mu\left(A_{n}\right) \rightarrow 0$ with $2 S\left(\chi_{A_{n}}\right) \geqslant \chi_{B_{n}} \cdot S 1$. Since $S$ is compact it follows that $\left\|S \chi_{A_{n}}\right\|_{\infty} \rightarrow 0$, i.e. $\chi_{B} S=0$.

5.4. Remark. If $E$ and $F$ are Köthe function spaces one might ask if one can replace in (C) the $L_{1}$-boundedness of the sequences $f_{n} \in E_{A}$ by $E$-boundedness. In general the answer is no: Every continuous linear operator $T: L_{p}(X, \mathscr{A}, \mu) \rightarrow$ $L_{p}(Y, \mathscr{B}, \nu), 2<p<\infty$, would have this modified property $(\mathrm{C})$. (Indeed, by Theorem 2 and Corollary 5 in [18], for a sequence $f_{n} \in L_{p}, 2<p<\infty$, with $\left\|f_{n}\right\|_{L_{p}}=1$, $f_{n} \stackrel{n}{\rightarrow} 0$ the following statements are equivalent:

(i) $f_{n} \stackrel{\mu}{\rightarrow} 0$,

(ii) every subsequence of $\left(f_{n}\right)$ has a subsequence equivalent to the unit vector basis of $l_{p}$,

(iii) $\left(f_{n}\right)$ has no subsequences equivalent to the unit vector basis of $l_{2}$, and it is impossible that $T$ map a basic sequence equivalent to $l_{p}$ into a basic sequence equivalent to $l_{2}$ for $p>2$.)

6. Operators with an atomic representation. Before giving characterizations analogous to 5.3 (in $6.5,6.6$ ) we name a class of 'typical' examples in $L_{a}(E, F)$ (compare 6.2).

6.1 Definition. Let $E$ and $F$ be Riesz function spaces over $(X, \mathscr{A}, \mu)$ and $(Y, \mathscr{B}, \nu)$, respectively. A linear operator $T: E \rightarrow F$

(a) is called a local Riesz homomorphism, if there are pairwise disjoint $A_{i} \in \mathscr{A}$ with $\cup_{i \in \mathrm{N}} A_{i}=X$ such that $T \chi_{A_{i}}$ is a Riesz homomorphism for all $i$,

(b) has the local Maharam property if there are pairwise disjoint $B_{i} \in \mathscr{B}$ with $\bigcup_{i \in \mathrm{N}} B_{i}=Y$ such that $\chi_{B_{i}}|T|$ has the Maharam property for all $i$.

Proposition 5.1 shows that these notions are dual to each other and indicates how such operators can be represented. The next theorem is due to N. J. Kalton ((i) $\Leftrightarrow$ (ii) is given in $[19, \S 3]$ with a different proof using the Kuratowski-RyllNardzewski selection theorem; (ii) $\Leftrightarrow$ (iii) is implicit in $[20, \S 5$; 19, Theorem 5.4]; the 'locally elementary operators' of [20] correspond to the 'local Riesz homomorphisms').

6.2 TheOREM. Let $E$ and $F$ be as in 6.1 with $1 \in E^{\times}, 1 \in F^{\times}$. For an order-continuous operator the following are equivalent.

(i) Thas an atomic representation. 
(ii) There are Borel functions $a_{n}: Y \rightarrow \mathbf{R}, \sigma_{n}: Y \rightarrow X$ such that for all $n \in \mathbf{N}$ and $\nu$-almost all $y \in Y$

$$
\begin{aligned}
& \left|a_{n}(y)\right| \geqslant\left|a_{n+1}(y)\right|, \quad \sum_{n=1}^{\infty}\left|a_{n}(y)\right|<\infty, \\
& \sigma_{n}(y) \neq \sigma_{m}(y) \quad \text { for } m \neq n
\end{aligned}
$$

and for all $f \in E$

$$
T f(y)=\sum_{n=1}^{\infty} a_{n}(y) f\left(\sigma_{n}(y)\right) \quad \nu \text {-a.e. }
$$

(iii) There is an increasing sequence of local Riesz homomorphisms $T_{n}$ : $E \rightarrow F$ such that $|T|=\sup _{n} T_{n}$.

(iv) There is an increasing sequence of operators $S_{n}: F^{\times} \rightarrow E^{\times}$with the local Maharam property such that $|T|^{\prime}=\sup S_{n}$.

As an immediate consequence of 6.2(iii), (iv) and 2.6 we obtain

6.3 Corollary. (a) The band generated by all Riesz homomorphisms in $L_{0}(E, F)$ equals the class of all operators with an atomic representation.

(b) The band generated by all operators with the Maharam property in $L_{0}(E, F)$ equals the class of all operators whose duals have an atomic representation.

The band generatd by a single Riesz homomorphism (or operator with the Maharam property) was studied in [31].

Proof of 6.2 . (a) $\Rightarrow$ (b). Since $(X, \mathscr{A}, \mu)$ is a standard measure space we may assume $X=[0,1] . M_{a}(X)$ denotes the space of all atomic measures on $X$ and let $\mathscr{B}^{*}$ be the Borel structure on $M_{a}(X)$ generated by the $w^{*}$-topology. Define

$$
\begin{aligned}
& a: M_{a}(X) \rightarrow \mathbf{R}, \quad a(\lambda)=\max _{x \in X}|\lambda(x)|, \\
& \phi: M_{a}(X) \rightarrow X, \quad \phi(\lambda)=\min \{x \in X:|\lambda(x)|=a(\lambda)\} .
\end{aligned}
$$

For $A_{i}^{n}=\left[(i-1) / 2^{n}, i / 2^{n}\right], i=1, \ldots, 2^{n}, n \in \mathbf{N}$, we have

$$
a(\lambda)=\inf _{n} \max _{i=1}^{2^{n}}\left|\lambda\left(A_{i}^{n}\right)\right|
$$

This shows that $a$ is $\mathscr{B}^{*}$-measurable. From

$$
\phi^{-1}([x, 1])=\left\{\lambda \in M_{a}(X): a\left(\left.\lambda\right|_{[0, x)}\right)<a\left(\left.\lambda\right|_{[x, 1]}\right)\right\}
$$

it follows that $\phi$ is $\mathscr{B}^{*}$-measurable since $\{\cdots\}$ belongs to $\mathscr{B}^{*}$. Also $\lambda \rightarrow s(\lambda)=$ sign $\lambda(\phi(\lambda))$ is $\mathscr{B}^{*}$-measurable (cf. [8 3.9]). Given a random measure $\left(\mu_{y}\right)_{y \in Y}$ representing $T$ we put

$$
\begin{aligned}
& a_{1}(y)=s\left(\mu_{y}\right) a\left(\mu_{y}\right), \quad \sigma_{1}(y)=\phi\left(\mu_{y}\right), \\
& a_{2}(y)=a\left(\mu_{y}-a_{1}(y) \delta_{\sigma_{1}(y)}\right), \quad \sigma_{2}(y)=\phi\left(\mu_{y}-a_{1}(y) \delta_{\sigma_{1}(y)}\right) \\
& \ldots \ldots
\end{aligned}
$$

Continuing like this we obtain measurable $a_{n}: Y \rightarrow \mathbf{R}, \sigma_{n}: Y \rightarrow X$ such that $\mu_{y}=\sum_{n=1}^{\infty} a_{n}(y) \delta_{\sigma_{n}(y)} \nu$-a.e. 
(b) $\Rightarrow$ (c). By Egoroff's theorem there are $Y_{n} \in \mathscr{B}$ and $\left(m_{n}\right)_{n \in \mathrm{N}}$ with

$$
Y_{n} \subset Y_{n+1} \subset \cdots, \quad \nu\left(Y-\bigcup_{n} Y_{n}\right)=0, \quad Y_{n} \text { compact }
$$

$$
\left.a_{m}\right|_{Y_{n}} \text { and }\left.\sigma_{m}\right|_{Y_{n}} \text { are continuous for all } n \text {, }
$$

$$
\sup _{y \in Y_{n}} \sum_{k=m_{n}}^{\infty}\left|a_{k}(x)\right| \rightarrow 0 \text { for } n \rightarrow \infty \text {. }
$$

For $T_{n} f(y)=\left(\sum_{k=1}^{m_{n}}\left|a_{k}(y)\right| f\left(\sigma_{k}(y)\right)\right) \chi_{Y_{n}}(y)$ we get $T_{n}(f)(y) \nearrow|T| f(y) \nu$-a.e. for all $f \in E_{+}$and it remains to show that the $T_{n}$ are local Riesz homomorphisms. Since all $\left.\sigma_{k}\right|_{Y_{n}}, k=1, \ldots, m_{n}$, are continuous and $\sigma_{k}(y) \neq \sigma_{l}(y)$ for $k \neq l$ there is for every $x \in X$ an open neighborhood $A_{x}$ such that $\sigma_{1}^{-1}\left(A_{x}\right), \ldots, \sigma_{m_{n}}^{-1}\left(A_{x}\right)$ are pairwise disjoint. Define

$$
\begin{aligned}
& \omega_{x}(y)= \begin{cases}\sigma_{k}(y) & \text { if } y \in \sigma_{k}^{-1}\left(A_{k}\right), k=1, \ldots, m_{n}, \\
x & \text { otherwise, }\end{cases} \\
& a_{x}(y)= \begin{cases}a_{k}(y) & \text { if } y \in \sigma_{k}^{-1}\left(A_{k}\right), k=1, \ldots, m_{n}, \\
0 & \text { otherwise. }\end{cases}
\end{aligned}
$$

Then $T_{n} \chi_{A_{x}}(f)(y)=a_{x}(y) \cdot f\left(\omega_{x}(y)\right)$, i.e. $T_{n} \chi_{A_{x}}$ is a Riesz homomorphism. Choose countably many $\left(A_{x_{i}}\right)_{i \in \mathrm{N}}$ with $X=\bigcup_{i} A_{x_{i}}$ and put $A_{i}=A_{x_{i}}-\bigcup_{j=1}^{i-1} A_{x_{j}}$.

(c) $\Rightarrow$ (a) is clear since $L_{a}(E, F)$ is a band.

(c) $\Leftrightarrow$ (b) follows by duality from 5.1 .

In 6.5 and 6.6 operators with an atomic representation and their duals are characterized by another weak form of the disjointness preseving property of Riesz homomorphisms, but these conditions will be stronger than the $u$ - $\nu$-continuity considered in 5.2, 5.3.

6.4 Definition. Let $E$ and $F$ be Riesz function spaces over $(X, \mathscr{A}, \mu)$ and $(Y, \mathscr{B}, \nu)$, respectively. A linear operator $T: E \rightarrow F$ is called

(a) $\mu$-continuous, if for every $L_{1}(X, \mu)$-bounded sequence $f_{n} \in E$ with $f_{n} \stackrel{\mu}{\rightarrow} 0$ we have $T f_{n} \stackrel{\nu}{\rightarrow} 0$,

(b) $u$-continuous, if for every $L_{1}(X, \mu)$-bounded sequence $f_{n} \in E$ with $f_{n} \stackrel{u}{\rightarrow} 0$ we have $T f_{n} \stackrel{u}{\rightarrow} 0$.

Also compare the condition 6.5(D) with 5.3(D) and 5.1(D), and condition 6.6(D') with 5.3(D) and the Maharam property.

6.5 TheOREM. Let $E$ and $F$ be as in 6.4 with $1 \in E^{\times}, 1 \in F^{\times}$. For an order-continuous operator $T: E \rightarrow F$ the following are equivalent.

(A) $T$ has an atomic representation.

(B) If $\Sigma$ is a o-subalgebra of $\mathscr{A}$ with $\left.\mu\right|_{\Sigma}$ diffuse and $S: E_{\Sigma} \rightarrow F$ is an integral operator with $0 \leqslant S \leqslant|T|_{E_{\Sigma}}$ then $S=0$.

(C) For every $\varepsilon>0$ there is an $A \in \mathscr{A}, \mu\left(A^{c}\right) \leqslant \varepsilon$, such that $T \chi_{A_{A}}$ is $\mu$-continuous. 
(D) For all $\varepsilon>0$ there is $a B \in \mathscr{B}$ with $\nu\left(B^{c}\right) \leqslant \varepsilon$, such that for every $a>1$ and every $B_{n} \in \mathscr{B}_{B}$ with $B_{n} \stackrel{u}{\rightarrow} 0$, there is a sequence $A_{n} \in \mathscr{A}$ with $A_{n} \stackrel{u}{\rightarrow} 0$ such that $a \cdot|T| \chi_{A_{n}} \geqslant \chi_{B_{n}} \cdot|T| 1$.

Proof. $(A) \Rightarrow(C)$. It is clear that every Riesz homomorphism and every local Riesz homomorphism is $\mu$-continuous and that the class of $\mu$-continuous operators is closed in $L_{0}\left(L_{1}, L_{1}\right)$ with respect to the operator norm. But given some operator $T \in L_{a}(E, F)$, there is (by 6.2(iii) and Egoroff's theorem) for every $\varepsilon>0$ an $A \in \mathscr{A}, \mu\left(A^{c}\right) \leqslant \varepsilon$ and a sequence of local Riesz homomorphisms $T_{n}$ such that

$$
\left\||T| \chi_{A}-T_{n} \chi_{A}\right\|_{L_{1}}=\left\|\chi_{A}\left|T^{\prime}\right|-\chi_{A} T_{n}^{\prime}\right\|_{L_{\infty}}=\left\|\chi_{A}\left(|T|^{\prime} 1-T_{n}^{\prime} 1\right)\right\|_{L_{\infty}} \rightarrow 0, \quad n \rightarrow \infty .
$$

(C) $\Rightarrow$ (B). For an integral operator $S: E \rightarrow F$ there is an $A \in \mathscr{A}, \mu\left(A^{c}\right) \leqslant \varepsilon$, such that $S \chi_{A}$ extends to a compact operator $S_{1}: L_{1}\left(\Sigma_{A}, \mu\right) \rightarrow L_{1}(Y, \nu)$ (cf. [7, §V.2, Proposition 21]). By [2] there is a sequence $0 \leqslant f_{n} \in L_{1}\left(\Sigma_{A}, \mu\right) \cap E_{\Sigma}$ with $\left\|f_{n}\right\|=1$, $f_{n} \stackrel{\mu}{\rightarrow} 0$ and $\left\|S_{1} f_{n}\right\| \rightarrow\left\|S_{1}\right\|_{L_{1}}$. If $0 \leqslant S \leqslant|T|_{E_{\Sigma}}$ and $|T| \chi_{A}$ is $\mu$-continuous it follows that $S_{1} f_{n} \stackrel{\nu}{\rightarrow} 0$. The compactness of $S_{1}$ in $L_{1}\left(\Sigma_{A}, \mu\right)$ implies $\left\|S_{1}\right\|=\lim \left\|S_{1} f_{n}\right\|=0$, i.e. $S \chi_{A}=0$.

(B) $\Rightarrow$ (A). If $T$ is not atomic, the diffuse component $|T|^{d}$ of $|T|$ is not zero and by 4.2(b) there is a $\sigma$-subalgebra $\Sigma$ such that $S=\left.|T|^{d}\right|_{E_{\Sigma}}$ is an integral operator not equal to zero.

$(\mathrm{A}) \Rightarrow(\mathrm{D})$. By 6.2(c) there is a sequence of local Riesz homomorphisms such that $0 \leqslant S_{n} \nearrow|T|$. By Egoroff's theorem there is for every $\varepsilon>0, a>a_{1}>1$, a compact $B_{0} \subset Y$ with $\nu\left(B_{0}^{c}\right) \leqslant \varepsilon / 2$ and an $S=S_{n}$ such that

$$
a_{1} S 1 \geqslant \chi_{B_{0}} \cdot|T| 1 \text {. }
$$

Let $D_{i} \in \mathscr{A}, i \in \mathbf{N}$, be a sequence of pairwise disjoint sets such that $S \chi_{D_{i}}$ is a Riesz homomorphism of the form

$$
S \chi_{D_{i}}(f)(y)=a_{i}(y) f\left(\sigma_{i}(y)\right) \quad \nu \text {-a.e. }
$$

Since $\sum_{i=1}^{r} S \chi_{D_{i}} \nearrow S$ there is (again by Egoroff's theorem) a compact $B \subset B_{0}$, $\nu\left(B^{c}\right) \leqslant \varepsilon$, and an $r \in \mathbf{N}$ such that

$$
\begin{gathered}
\frac{a}{a_{1}} \cdot \sum_{i=1}^{r} S \chi_{D_{i}} \geqslant \chi_{B} \cdot S 1, \\
\left.a_{i}\right|_{B},\left.\sigma_{i}\right|_{B} \text { are continuous for all } i \in \mathbf{N} .
\end{gathered}
$$

Given $B_{m} \subset B, B_{m} \stackrel{u}{\rightarrow} 0$, we define $A_{m}=\bigcup_{i=1}^{r} \sigma_{i}\left(B_{m}\right)$. From (6.5), (6.6) and (6.4) it follows that

$$
\begin{aligned}
a|T| \chi_{A_{m}} & \geqslant a \sum_{i=1}^{r} S\left(\chi_{A_{m} \cap D_{i}}\right)=a \sum_{i=1}^{r} \chi_{\sigma_{i}^{-1}\left(A_{m}\right)} S\left(\chi_{D_{i}}\right) \\
& \geqslant a \cdot \chi_{B_{m}} \cdot \sum_{i=1}^{r} S\left(\chi_{D_{i}}\right)=a_{1} \chi_{B_{m}} S(1) \geqslant \chi_{B_{m}}|T| 1 .
\end{aligned}
$$


To see that $A_{m} \stackrel{u}{\rightarrow} 0$ choose a sequence $\left(m_{l}\right)$ such that $\cap C_{n}, C_{n}=\overline{\bigcup_{l=n}^{\infty} B_{m l}}$, is countable. Since for every $i=1, \ldots, r$

$$
D_{i} \cap \bigcap_{n} \overline{\bigcup_{l=n}^{\infty} A_{m_{l}}} \subset \bigcap_{n}\left[D_{i} \cap \overline{\overline{\bigcup_{i=1}^{r} \sigma_{i}\left(\bigcup_{l=n}^{\infty} B_{m}\right)}}\right] \subset \bigcap_{n} \sigma_{i}\left(C_{n}\right)=\sigma_{i}\left(\bigcap C_{n}\right)
$$

is countable (use (6.7)), it follows that $A_{n} \stackrel{u}{\rightarrow} 0$.

(D) $\Rightarrow$ (A). If $|T|$ has property (D) then so does the diffuse component $S$ of $|T|$. Given a $B$ as in (D), we choose sequences $B_{n} \subset B,\left(n_{m}\right)$ with

$$
d\left(B_{n}\right) \stackrel{n \rightarrow \infty}{\rightarrow} 0, \quad \bigcup_{n=n_{m}+1}^{n_{m+1}} B_{n}=B
$$

By (D) there is a sequence $A_{n} \in \mathscr{A}$ with

$$
A_{n} \stackrel{\mu}{\rightarrow} 0, \quad S \chi_{A_{n}} \geqslant \frac{1}{2} \chi_{B_{n}} \cdot S 1 .
$$

From (6.8) and (6.9) we obtain

$$
\chi_{B} \cdot S(1)=\varlimsup_{n} \chi_{B_{n}} S(1) \leqslant 2 \varlimsup_{n} S\left(\chi_{A_{n}}\right)=0
$$

since $A_{n} \stackrel{u}{\rightarrow} 0$ and $S$ has a diffuse representation by $4.2(\mathrm{c})$. Hence $\chi_{B} S=0$.

6.6 ThEOREM. Let $E$ and $F$ be as in 6.4 with $1 \in E^{\times}, 1 \in F^{\times}$. For an order-continuous operator $T: E \rightarrow F$ the following are equivalent.

(A) $T^{\prime}: F^{\times} \rightarrow E^{\times}$has an atomic representation.

(C') For every $\varepsilon>0$ there is an $A \in \mathscr{A}, \mu\left(A^{c}\right) \leqslant \varepsilon$, such that $T \chi_{A_{n}}$ is u-continuous.

(D') For every $\varepsilon>0$ there is a $B \in \mathscr{B}, \nu\left(B^{c}\right) \leqslant \varepsilon$, such that for every $a>1$ and every sequence $B_{n} \in \mathscr{B}_{B}$ with $\nu\left(B_{n}\right) \rightarrow 0$ there is a sequence $A_{n} \in \mathscr{A}$ with $\mu\left(A_{n}\right) \rightarrow 0$ and $a|T| \chi_{A_{n}} \geqslant \chi_{B_{n}}|T| 1$.

(E') For every $\varepsilon>0$ there is an $A \in \mathscr{A}, \mu\left(A^{c}\right)_{u} \leqslant \varepsilon$, such that for a martingale $\left(\mathscr{F}_{n}, \Sigma_{n}\right)$ determined by $T \chi_{A}$ we have $\mathscr{F}_{n}(x) \stackrel{u}{\rightarrow} 0 \mu$-a.e. in $A$.

Proof. (A) $\Rightarrow\left(\mathrm{C}^{\prime}\right)$. Applying 2.4 to $T^{\prime}$ gives an $A \in \mathscr{A}$ with

$$
A \text { compact, } \mu\left(A^{c}\right) \leqslant \varepsilon,\left.\quad \mu\right|_{A} \text { strictly positive, }
$$

$$
|T| \chi_{A}: L_{1}(A, \mu) \rightarrow L_{1}(Y, \nu) ; \quad|T|_{\chi_{A}}: M(A) \rightarrow M(Y) \text { is } w^{*}-
$$
continuous and $|T|\left(U_{M(A)}\right)$ is uniformly tight,

$$
\nu_{x}=|T| \chi_{A}\left(\delta_{x}\right) \quad \text { is atomic for all } x \in A .
$$

Let $\left(f_{n}\right)$ be a sequence with $\left\|f_{n}\right\|_{L_{1}(A, \mu)} \leqslant 1$ and $f_{n} \stackrel{u}{\rightarrow} 0$. In order to show that $T f_{n} \stackrel{u}{\rightarrow} 0$ we may assume, in addition, that $\left(\left|f_{n}\right| \cdot \mu\right)_{n \in \mathbf{N}} w^{*}$-converges to some $\lambda \in M(A)$. Since $\left|f_{n}\right| \cdot \mu \stackrel{u}{\rightarrow} 0$ it follows from 3.4 that $\lambda$ is an atomic measure of the form $\lambda=\sum_{i=1}^{\infty} \alpha_{i} \delta_{x_{i}}$ where $\sum_{i=1}^{\infty}\left|\alpha_{i}\right|<\infty$ and $x_{i} \in A$. Then by (6.12),

$$
|T| \lambda=\sum_{i=1}^{\infty} \alpha_{i}|T|\left(\delta_{x_{i}}\right)=\sum_{i=1}^{\infty} \alpha_{i}\left|\nu_{x_{i}}\right|
$$


is an atomic measure and from $|T|\left(f_{n}\right) \cdot \stackrel{w^{*}}{\rightarrow}|T| \lambda$ together with 3.4 it follows that $\left|T f_{n}\right| \leqslant|T|\left(\left|f_{n}\right|\right) \stackrel{u}{\rightarrow} 0$.

$\left(C^{\prime}\right) \Rightarrow\left(E^{\prime}\right)$ is proved similarly to $(C) \Rightarrow(E)$ of 5.3 (also the proof of 5.3, $(\mathrm{A}) \Rightarrow(\mathrm{E})$ can be adapted to the present situation by using 3.4).

$\left(\mathrm{E}^{\prime}\right) \Rightarrow(\mathrm{A})$. Let $\left(\mathscr{F}_{n}, \Sigma\right)$ and $A$ be as in $\left(E^{\prime}\right)$. By 2.8 there is an $A_{0} \subset A$, $\mu\left(A-A_{0}\right)=0$, such that $\mathscr{F}_{n}(x) \cdot \nu \stackrel{w^{*}}{\rightarrow} \nu_{x}$ for all $x \in A_{0}$. If $\lambda_{x}$ is a $w^{*}$-limit of $\left|\mathscr{F}_{n}(x)\right| \cdot \nu$, then $\left|\nu_{x}\right| \leqslant \lambda_{x}$ for $x \in A_{0}$. Since $\left|\mathscr{F}_{n}(x)\right| \cdot \mu \stackrel{u}{\rightarrow} 0$ it follows from 3.4 that $\lambda_{x}$ and $\nu_{x}$ are atomic for $x \in A_{0}$.

$(\mathrm{A}) \Rightarrow\left(\mathrm{D}^{\prime}\right)$. By 6.2 (iv) there is a sequence of operators $S_{n}: E \rightarrow F$ with the local Maharam property such that $0 \leqslant S_{n} \nearrow|T|$. For every $\varepsilon>0$ and $a>1$ there is a $B_{0} \subset Y$ with $\nu\left(B_{0}^{c}\right) \leqslant \varepsilon / 2$ and an $S=S_{n}$ with

$$
a \chi_{B_{0}} \cdot S(1) \geqslant \chi_{B_{0}}|T|(1) \text {. }
$$

By Definition 6.1 there is a $B \subset B_{0}, \nu\left(B^{c}\right) \leqslant \varepsilon$, and finitely many pairwise disjoint $C_{1}, \ldots, C_{r}$ such that $B=C_{1} \cup \cdots \cup C_{r}$ and all $\chi_{C_{i}} S, i=1, \ldots, r$, are operators with the Maharam property. By 5.1 there are $\nu$-orthogonal random measures $\left(\nu_{y}^{(i)}\right)_{y \in C_{i}}$ with Borel maps $\sigma_{i}: X \rightarrow C_{i}$ and measurable maps $b_{i}: X \rightarrow \mathbf{R}$ such that for all $f \in E$

$$
\chi_{C_{i}} S(f)(y)=\int_{\sigma_{i}^{-1}(y)} f \cdot b_{i} d \nu_{y}^{(i)} \quad \nu \text {-a.e. in } C_{i} .
$$

Given $B_{n} \subset C_{i}$ it follows from (6.14) that $A_{n}=\sigma_{i}^{-1}\left(B_{n}\right) \cap\left\{b_{i}>0\right\}$ satisfies

$$
\chi_{C_{i}} S\left(\chi_{A_{n}}\right)(y)=\chi_{B_{n}} S(1)(y) \quad \nu \text {-a.e. in } C_{i} \text {. }
$$

If $\nu\left(B_{n}\right) \rightarrow 0$ then $\mu\left(A_{n}\right) \rightarrow 0$ because

$$
\int_{B_{n}} S(1) d \nu=\int \chi_{C_{i}} \cdot S\left(\chi_{A_{n}}\right) d \nu=\int_{A_{n}} S^{\prime}\left(\chi_{C_{i}}\right) d \mu .
$$

For general $B_{n} \subset B$ we put $A_{n}=\bigcup_{i=1}^{r} A_{n, i}, A_{n, i}=\sigma_{i}^{-1}\left(B_{n}\right) \cap\left\{b_{i}>0\right\}$. (6.13) and (6.15) gives

$$
S\left(\chi_{A_{n}}\right) \geqslant \sup _{i=1}^{r} S\left(\chi_{A_{n, i}}\right) \geqslant \chi_{B_{n}} \cdot S 1 \geqslant a^{-1} \cdot \chi_{B_{n}}|T|(1) .
$$

$\left(\mathrm{D}^{\prime}\right) \Rightarrow(\mathrm{A})$. Let $B$ be as in $\left(\mathrm{D}^{\prime}\right)$. For every $\sigma$-subalgebra $\Sigma \subset \mathscr{B}_{B}$ one can show, as in 5.3, (D) $\Rightarrow(\mathrm{B})$, that every integral operator $E \rightarrow F_{\Sigma}$ with $0 \leqslant S \leqslant \mathscr{E}_{\Sigma}\left(\chi_{B}|T|\right)$ is zero. By duality every integral operator $S^{\prime}: F_{\Sigma}^{\times} \rightarrow E^{\times}$with $0 \leqslant S^{\prime} \leqslant\left(\chi_{B}|T|\right)^{\prime}$ is zero and $\left(\chi_{B}|T|\right)^{\prime}$ has an atomic representation.

\section{REFERENCES}

1. W. Arveson, Operator algebras and invariant subspaces, Ann. of Math. (2) 100 (1974), 433-532.

2. G. R. Barnes and R. Whitley, The oscillation of an operator on $L_{p}$, Trans. Amer. Math. Soc. 211 (1975), 339-351.

3. J. Bourgain, Dunford-Pettis operators on $L^{1}$ and the Radon-Nikodym property, Israel J. Math. 37 (1980), 34-47.

4. J. R. Brown, Approximation theorems for Markov operators, Pacific J. Math. 16 (1966), 12-23.

5. A. Buhvalov, Integral representation of linear operators, J. Soviet Math. 9 (1978), 129-137.

6. M. Capon, Primarité de $l_{p}\left(L_{1}\right)$, Math. Ann. 250 (1980), 53-63.

7. J. Diestel and J. Uhl, Vector measures, Math. Surveys, no. 15, Amer. Math. Soc., Providence, R. I., 1977. 
8. R. Doss, Convolution of singular measures, Studia Math. 45 (1973), 111-117.

9. L. Dubins and D. Freedman, Measurable sets of measures, Pacific J. Math. 14 (1964), 1211-1223.

10. N. Dunford and B. J. Pettis, Linear operations on summable functions, Trans. Amer. Math. Soc. 47 (1940), 323-392.

11. P. Enflo and T. Starbird, Subspaces of $L_{1}$ containing $L_{1}$, Studia Math. 65 (1979), 203-225.

12. H. Fakhoury, Representations d'opérateurs a valeurs dans $L^{1}(X, \Sigma, \mu)$, Math. Ann. 240 (1979), 203-212.

13. S. Foguel, The ergodic theory of Markov' processes, Van Nostrand Reinhold, New York, 1969.

14. D. H. Fremlin, A positive compact operator, Manuscripta Math. 15 (1975), 323-327.

15. C. C. Graham and O. C. McGehee, Essays in commutative harmonic analysis, Springer-Verlag. Berlin, Heidelberg and New York, 1979.

16. C. Ionescu Tulcea, Sur certaines endomorphismes de $L_{c}^{\infty}(Z, \mu)$, C. R. Acad. Sci. Paris Ser. 261 (1965), 4961-4963.

17. W. B. Johnson and L. Jones, Every $L_{p}$-operator is an $L_{2}$-operator, Proc. Amer. Math. Soc. 72 (1978), 309-312

18. M. Kadec and A. Pelczyńnki, Basis, lacunary sequences and complemented subspaces in the spaces $I_{. p}$, Studia Math. 21 (1962), 161-176.

19. N. J. Kalton, The endomorphisms of $L_{p}(0 \leqslant p \leqslant 1)$, Indiana Univ. Math. J. 27 (1978), 353-381.

20. L__ Linear operators on $L_{p}$ for $0 \leqslant p \leqslant 1$. Trans. Amer. Math. Soc. 259 (1980), 317-355.

21. __ Embedding $L_{1}$ in a Banach lattice, Israel J. Math. 32 (1979), 209-220.

22. J. L. Kelly, General topologv, Van Nostrand, New York, 1955.

23. M. A. Krasnoselskii, P. P. Zabreiko, E. I. Pustylnik and E. B. Sbolevskii, Integral operators in spaces of summable functions, Nordhoff, Leyden, 1976.

24. S. Kwapien, On the form of a linear operator in the space of all measurable functions, Bull. Acad. Polon. Sci. Sér. Sci. Phys. Astronom. 21 (1973), 951-954.

25. H. E. Lacey, The isometric theory of classical Banach spaces, Springer-Verlag, Berlin, Heidelberg and New York, 1974.

26. J. Lindenstrauss and L. Tzafriri, Classical Banach spaces. I. Sequence spaces, Springer-Verlag, Berlin, Heidelberg and New York, 1977.

27. Classical Banach spaces. II. Function spaces, Springer-Verlag, Berlin, Heidelberg and New York, 1979 .

28. H. Lotz, Extensions and liftings of positive linear mappings on Banach lattices, Trans. Amer. Math. Soc. 211 (1975), 85-100.

29. W. A. J. Luxemburg and A. C. Zaanen. The linear modulus of an order bounded linear transformation, Indag. Math. 33 (1971), 422-447.

30. Riesz spaces, North-Holland, Amsterdam and London, 1971.

31. W. A. J. Luxemburg and A. R. Schep, A Radon-Nikodym type theorem for positive operators and a dual, Nederl. Akad. Wetensch. Proc. Ser. A 81 (1978).

32. W. A. J. Luxemburg, Some aspects of the theory of Riesz spaces, University Arkansas Lecture Notes in Math., Fayetteville, Ark., 1979.

33. R. D. Mauldin, D. Preiss and H. v. Weizsäcker, Orthogonal transition kernels, Ann. Probab. (to appear).

34. R. J. Nagel and U. Schlotterbeck, Integraldarstellung regulärer Operatoren auf Banachierbänden. Math. Z. 127 (1972), 293-300.

35. H. Neunzert, An introduction to nonlinear Boltzmann-Vlasov equation, Lecture Notes Internat. Summer School, Montecatini, 1981.

36. J. Neveu, Bases mathématiques du calcul des probabilites, Masson, Paris, 1964

37. K. R. Parthasarathy, Probability measures on metric spaces, Academic Press, New York and London. 1967

38. A. Pelczyński, Strictly singular and strictly cosingular operators in $L_{1}(\nu)$-spaces, Bull. Acad. Polon. Sci. 13 (1965), 37-41.

39. H. H. Schaefer, Banach lattices and positive operators, Springer-Verlag, Berlin, Heidelberg and New York, 1974

40. A. R. Schep, Kernel operators, Nederl. Akad. Wetensch. Proc. Ser. A 82(1) (1979), 39-53.

41. A. R. Sourour, Pseudo-integral operators, Trans. Amer. Math. Soc. 253 (1979), 339-363.

42. . Characterization and order properties of pseudo-integral operators, Pacific J. Math. 99 (1982),

$145-159$ 
43. T. Starbird, Subspaces of $L_{1}$ containing $L_{1}$, Ph.D. thesis, University of California, Berkeley, 1976.

44. B. Z. Vulikh, Introduction to the theory of partially ordered spaces, Wolters-Nordhoff, Groningen, 1967.

45. L. Weis, Integral operators and changes of density, Indiana Univ. Math. J. 31 (1982), 83-96.

46. __ A note on diffuse random measures, Z. Wahrsch. Verw. Gebiete 65 (1983), 239-244.

47. Decompositions of positive operators and some of their applications, Functional Analysis: Surveys and Recent Results. III (Proc. 3rd Paderborn Conf. Functional Anal.), North-Holland, Amsterdam, 1984.

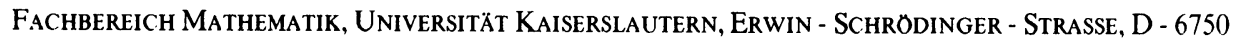
KAISERSLAUTERN, WeSt GermanY 